\title{
EVERY CONTRACTIBLE FAN IS LOCALLY CONNECTED AT ITS VERTEX
}

BY

\author{
LEX G. OVERSTEEGEN ${ }^{1}$
}

\begin{abstract}
We prove that each contractible fan is locally connected at its vertex. It follows that every contractible fan is embeddable in the plane. This gives a solution to a problem raised by J. J. Charatonik and C. A. Eberhart.
\end{abstract}

1. Introduction. It is an open question to characterize those fans which are embeddable in the plane (for an example of a fan which is not embeddable in the plane, see [2]). In another paper [10], the author has shown that every fan, which is locally connected at the vertex, can be embedded in the plane. The main purpose of this paper is to show that each contractible fan is locally connected at its vertex. Hence, combining the results above, it follows that every contractible fan is embeddable in the plane. This gives a solution to a problem raised by $J$. $J$. Charatonik and C. A. Eberhart [3, Problem 788].

The concept of contractibility is well known in general topology. However, even for very simple spaces, it is often quite difficult to prove that a space is noncontractible. In particular, for a rather limited class of spaces, namely dendroids, no internal characterization of contractibility is known.

The results of this paper will be used in a forthcoming paper [12], to give an internal characterization of contractibility for fans, and to give a solution to a problem raised by D. P. Bellamy and J. J. Charatonik in [1].

The main result of this paper is the proof that each contractible fan is locally connected at its vertex. The proof of this fact is quite complicated. The main steps of the proof are outlined in Example 2.3 and Remark 2.4.

2. Definitions and preliminaries. By a continuum we mean a compact connected metric space. A dendroid is a hereditarily unicoherent and arcwise connected continuum. By a fan we understand a dendroid which has exactly one branchpoint, and we call this point the vertex of the fan $X$. If $x, y$ are points in a dendroid $X$, then we denote by $[x, y]$ the unique arc in $X$ having $x$ and $y$ as endpoints. The weak-cut order $\leqslant$, with respect to a point $p$ in a dendroid $X$, is given by $x<y$ if and only if $[p, x] \subset[p, y]$. A space $X$ is contractible provided there exists a continuous transformation $H: X \times I \rightarrow X$ such that $H(x, 0)=x$ for each $x \in X$ and $H(X \times\{1\})$ is one point.

Received by the editors June 14, 1978 and, in revised form, June 12, 1979. Presented to the Twelfth Spring Topology Conference at Norman, Oklahoma, March 1978.

AMS (MOS) subject classifications (1970). Primary 54F20, 54E40; Secondary 54F50, 54F55.

${ }^{1}$ This research is part of the author's doctoral dissertation, completed at Wayne State University, Detroit, Michigan. 
A dendroid $X$ is of type $N$ (cf. [9]) if there exists an arc $A \subset X$ with endpoints $p$ and $q$, two sequences of arcs $A_{i} \subset X$ and $B_{i} \subset X(i=1,2, \ldots)$ with endpoints $p_{i}$, $p_{i}^{\prime}$ and $q_{i}, q_{i}^{\prime}$ respectively; and points $q_{i}^{\prime \prime} \in A_{i} \backslash\left\{p_{i}, p_{i}^{\prime}\right\}$ and $p_{i}^{\prime \prime} \in B_{i} \backslash\left\{q_{i}, q_{i}^{\prime}\right\}$ such that the following conditions are satisfied: (1) $A=\operatorname{Lim} A_{i}=\operatorname{Lim} B_{i}$, (2)p $p=\lim p_{i}$ $=\lim p_{i}^{\prime}=\lim p_{i}^{\prime \prime}$, (3) $q=\lim q_{i}=\lim q_{i}^{\prime}=\lim q_{i}^{\prime \prime}$. The following theorem was proved in [9, Corollary 2.2].

\subsection{TheOREM. If $X$ is a dendroid of type $N$, then $X$ is noncontractible.}

A fan $X$ has property $P$ (cf. [10]) provided for each sequence $\left\{v_{i}\right\}$ in $X$ converging to the vertex $v$ of $X$ we have $\operatorname{Ls}\left[v, v_{i}\right]=\{v\}$. We will use the following result which was proved in [10, Theorem 3.1].

2.2. ThEOREM. Let $X$ be a fan with vertex $v$, then the following are equivalent.

(i) $X$ has property $P$.

(ii) $X$ is locally connected at $v$.

If $p$ and $q$ are points in the plane, we denote by $\overline{p q}$ the straight line closed segment with endpoints $p$ and $q$.

2.3. ExAmple. Let $v=(0,1), a=(0,0), a(i, 1)=(1 / i, 0), a(i, 2)=(-1 / i, 0)$ and $v_{i}=(-1 / i, 1)$. Denote by $S_{i}$ the semicircle $S_{i}=\left\{(x, y) \mid x^{2}+y^{2}=1 / i^{2}, y<\right.$ $0\}$, joining the points $a(i, 1)$ and $a(i, 2)$ for $i=1,2, \ldots$ Then

$$
X=\overline{a v} \cup \bigcup_{i=1}^{\infty}\left(\overline{v a(i, 1)} \cup S_{i} \cup \overline{v_{i} a(i, 2)}\right)
$$

is a noncontractible fan.

Proof. Observe that $X$ is a fan of type $N$ and hence, by Theorem $2.1, X$ is noncontractible. Since in general it is not true that there exists a sequence $\left\{v_{i}\right\} \rightarrow v$ such that $\operatorname{Ls}\left[v, v_{i}\right]=K \neq\{v\}$, where $K$ is an arc, we will show in different manner that $X$ is noncontractible. The proof in the following pages follows the same main steps (see also Remark 2.4).

Suppose $X$ is contractible and let $H: X \times I \rightarrow X$ be a contraction. Let $z=$ $(0,1 / 2) \in X$ and let $\delta>0$ be such that $d\left(H(x, t), H\left(x^{\prime}, t^{\prime}\right)\right)<1 / 4$ if $d\left((x, t),\left(x^{\prime}, t^{\prime}\right)\right)<3 \delta$. Let $\left\{x_{i}\right\}_{i=1}^{n}$ be a sequence in $K \backslash B(v, \delta)$ such that $x_{1}=$ $\operatorname{Bd}(B(v, \delta)) \cap[a, v], x_{i}<x_{i+1}(i=1, \ldots, n-1), x_{n}=a, x_{c}=z$ and $d\left(x_{i}, x_{i+1}\right)$ $<\delta / 4(i=1, \ldots, n-1)$. Put

$$
\alpha=\min \left\{1 / 4,1 / 5 \delta, \min _{i \neq j} d\left(x_{i}, x_{j}\right)\right\} / 3 .
$$

Let $U_{j}=B\left(x_{j}, \alpha / 2\right)$ and let $K(i, 0)$ denote the component of $\left[v, v_{i}\right] \backslash B(v, \alpha / 2)$ containing $a(i, 1)$. Observe that, for $i>N>0, K(i, 0) \cap \bar{U}_{j}$ consists of exactly two components. In each component of $\bar{U}_{j} \cap K(i, 0), 1<j<n$, choose one point $x(i, 0, j, w), w \in\{1,2\}$, such that $x(i, 0, j, 1)>x(i, 0, j, 2)$. Put $x(i, 0, n, 1)=$ $a(i, 2), x(i, 0, n, 2)=a(i, 1), z(i, 1)=x(i, 0, c, 2)$ and $z(i, 2)=x(i, 0, c, 1)$.

Since $H(\{v\} \times I)$ is a locally connected continuum, there exists $i_{0}>N$ such that $z\left(i_{0}, 1\right) \notin H(\{v\} \times I)$ and $d\left(a\left(i_{0}, 1\right), a\left(i_{0}, 2\right)\right)<\delta / 2$. Moreover we may assume that $H(X \times\{1\}) \neq z\left(i_{0}, 1\right)$. From here on we will take $i=i_{0}$ and omit the 
subscript $i_{0}$. Let $C$ be the component of $H^{-1}(z(1))$ in $[v, a(1)] \times I$ containing the point $(z(1), 0)$. Then

$$
C \cap[(\{v\} \times I) \cup(X \times\{1\})]=\varnothing .
$$

It follows (see $\S 6$, Claim 6) that $C \cap(\{a(1)\} \times I) \neq \varnothing$. Put $y_{j}=x(0, j, 2)$ and $p_{j}=x(0, j, 1)$; hence $y_{c}=z(1)$ and $y_{n}=a(1)$. There exists (cf. Lemma 4.1 and Corollary 4.2) a function $F$, a number $r$ and a sequence $\left\{t_{k}\right\}$ in $I$ such that

(1) $F:\{1,2, \ldots, r\} \rightarrow\{1,2, \ldots, n\}$,

(2) $\left(y_{F(1)}, t_{1}\right)=(z(1), 0)$ and $y_{F(r)}=a(1)$, hence $F(1)=c$ and $F(r)=n$,

(3) $d\left(\left(y_{F(k)}, t_{k}\right), C\right)<\delta / 3, k=1, \ldots, r$,

(4) $d\left(\left(y_{F(k)}, t_{k}\right),\left(y_{F(k+1)}, t_{k+1}\right)\right)<\delta / 3$.

Let $W$ be the component of $\left[v, v_{i_{0}}\right] \cap B(z, 1 / 4)$ in $X$, containing the point $z(2)$. By "copying" the sequence $\left\{\left(y_{F(k)}, t_{k}\right)\right\}$ in $\left[a(2), v_{i_{0}}\right]$, i.e. by considering the sequence $\left\{\left(p_{F(k)}, t_{k}\right)\right\}$, it follows easily by induction (see also Lemma 5.6) that $H\left(p_{F(r)}, t_{r}\right)=H\left(a(2), t_{r}\right) \in W$.

Hence $\operatorname{diam}\left(H\left([a(1), a(2)] \times\left\{t_{r}\right\}\right)\right)>1 / 3$. But $\operatorname{diam}\left([a(1), a(2)] \times t_{r}\right)<\delta$ and hence $\operatorname{diam}\left(H\left([a(1), a(2)] \times t_{r}\right)\right)<1 / 4$. This contradiction completes the proof.

2.4. REMARK. The proof that each contractible fan is locally connected at its vertex, or, equivalently, has property $P$, follows the same main steps as the proof above. The difference is that the continuum $K=\operatorname{Ls}\left[v, v_{i}\right] \neq\{v\}$ may be a (nondegenerate) locally connected fan. In this case the "copying procedure", outlined above, becomes much more involved. In $\$ 6,(8)-(10)$, a sequence $\left\{x_{j}\right\}_{j=1}^{n}$ is constructed similar to the one above. This sequence, using Lemmas 5.1-5.5, provides a labeling of points on the $\operatorname{arcs}\left[v, v_{i}\right]$. Using Lemma 4.1 and Corollary 4.2 we construct a function $F$ satisfying properties like (1)-(4) above. In $\$ 3$ we investigate under which conditions a "copying procedure" is possible. In $\S 6$, Claims 2-4 and 7-8, we verify that these conditions hold and, using Lemma 5.6, arrive at the same contradiction as above.

The following combinatorial lemma seems related to the concept of uniformization of functions (see [8]), which was introduced to study some properties of the pseudoarc.

3. A combinatorial lemma. The following lemma (see Lemma 3.1) is used in the proof of Theorem 6.1, where we prove that every contractible fan is locally connected at its vertex. Since this is the only use of this lemma, we did not try to give it the strongest possible formulation. On the contrary, we chose to formulate it in such a way that the conditions can be easily checked later and that its proof is almost trivial, although still rather messy. We start with some notation needed in Lemma 3.1.

In this section we will denote by $N$ the set of natural numbers with ordering $<$. All functions will be functions of subsegments of $N$ into $N$. If $f:[a, b] \rightarrow N$ is a function, then we say that $x \in[a, b]$ is a local maximum provided that if $|x-y|<$ 1 and $y \in[a, b]$, then $f(y)<f(x)$.

Let $f:[1, p] \rightarrow N$ and $g:[1, q] \rightarrow N$ be two functions. Then we say that $f$ and $g$ are uniform of order $n$ provided the following two conditions are satisfied. 
(1) For each number $t, 0<t \leqslant n$, there exists a number $k(t)>0$ and a sequence $t=a_{0}>a_{1}>\cdots>a_{k(t)}=0$ such that

(i) if $i-1, i \in[1, p](j-1, j \in[1, q])$ and $f(i-1)<f(i)=t(g(j-1)<g(j)$ $=t)$, then $f(i-s)=a_{s}\left(g(j-s)=a_{s}\right)$ for each $s \in[0, k(t)]$,

(ii) if $i+1, i \in[1, p](j+1, j \in[1, q])$ and $f(j+1)<f(j)=t(g(j+1)<g(j)$ $=t$, then $f(i+s)=a_{s}\left(g(j+s)=a_{s}\right.$, respectively) for $s \in[0, k(t)]$.

(2) For each $i \in[1, p](j \in[1, q])$ such that $f(i)>0(g(j)>0)$ at least one of the following two statements is true.

(i) $i+1 \in[1, p](j+1 \in[1, q])$ and $f(i+1)<f(i)(g(j+1)<g(j))$,

(ii) $i-1 \in[1, p](j-1 \in[1, q])$ and $f(i-1)<f(i)(g(j-1)<g(j)$, respectively).

We will say that the sequence $\left\{a_{s}\right\}(s=0,1, \ldots, k(t))$ is associated with the number $t$.

Let $f:[1, r] \rightarrow N$ be a function, $n=\max \{f(k) \mid k \in[1, r]\}, t_{0}=\max \{f(k) \mid k \in$ $[1, r]$ and $f(k)<n\}$ and $k_{0}=\max \left\{k \in[1, r] \mid f(k)=t_{0}\right\}$. If $f(r)<n$ and $k_{0} \neq r$, then we define a sequence $\left\{\left(t_{v}, k_{v}\right)\right\}$ by induction. Suppose $t_{v-1}$ and $k_{v-1}$ have been defined and $k_{v-1} \neq r$. Let $t_{v}=\max \left\{f(k) \mid k_{v-1}<k<r\right\}$ and $k_{v}=\max \{k \in$ $\left.[1, r] \mid f(k)=t_{v}\right\}$. The sequence $\left\{\left(t_{v}, k_{v}\right)\right\}\left(v=0,1, \ldots, \gamma, f(r)=t_{\gamma}\right)$ will be called the local maxima sequence of $f$. Notice that if $f(r)=n$ or if $k_{0}=r$, then the local maxima sequence of $f$ reduces to a single pair $\left\{\left(t_{0}, k_{0}\right)\right\}$.

Let $f:[1, r] \rightarrow N$ and $g:[1, q] \rightarrow N$ be two functions such that $\max \{f(k) \mid k \in$ $[1, p]\}=\max \{g(j) \mid j \in[1, q]\}=n$.

Let $s$ be a point of $[1, q]$ such that $g(s)=f(r)$. Then we say that $f$ and $g$ are symmetric with respect to the points $r$ and $s$ provided that if $\left\{\left(t_{v}, k_{v}\right)\right\}(v=$ $0,1, \ldots, \gamma)$ is the local maxima sequence of $f$ and if we put $t_{-1}=n, k_{-1}=1$, then

(3) for each $v \in[-1, \gamma]$, there exists $j_{v} \in[1, q]$, where we take $j_{-1}=1$, such that

(i) $g\left(j_{v}\right)=t_{v}$ and $g(j)<t_{v}$ for every $j \in\left(j_{v}, s\right)$,

(ii) for each $k \in\left(k_{v}, r\right)$ there exists $j^{*} \in[1, q]$ such that $f(k)=g\left(j^{*}\right)$ and $g(j)<t_{v}$ for every $j \in\left(j^{*}, s\right)$,

(iii) for each $j \in\left(j_{v}, s\right)$ there exists $k \in\left[k_{v}, r\right]$ such that $f(k)=g(j)$.

3.1. Lemma. Let $P=[1, p]$ and $Q=[1, q]$, and $f$ and $g$ be functions of $P$ and $Q$ into $N$, respectively. Let $R=[1, r], F: R \rightarrow P$ a function, $s$ a point of $Q$ and $n \in N$ such that

(4) $f(1)=g(1)=n, f(i) \leqslant n-1$ if $i \in(1, p), g(j)<n-1$ if $j \in(1, q), f(p)<n$ and $g(q)<n$.

(5) $F(1)=1, f \circ F(k)<n-1$ if $k \in(1, r)$ and $f \circ F(r)=g(s)$.

(6) $|F(k+1)-F(k)| \leqslant 1$ if $k \in[1, r-1]$.

(7) $f$ and $g$ are uniform of order $n$.

(8) If $i_{1}<i_{2}, i_{1}, i_{2} \in P\left(j_{1}<j_{2}, j_{1}, j_{2} \in Q\right)$ and there exists $i \in\left(i_{1}, i_{2}\right)(j \in$ $\left.\left(j_{1}, j_{2}\right)\right)$ such that $f(i)=0 \quad(g(j)=0)$, then for each natural number $t<$ $\min \left\{f\left(i_{1}\right), f\left(i_{2}\right)\right\}\left(t<\min \left\{g\left(j_{1}\right), g\left(j_{2}\right)\right\}\right)$ there exists $i_{3} \in\left(i_{1}, i_{2}\right)\left(j_{3} \in\left(j_{1}, j_{2}\right)\right)$ with $f\left(i_{3}\right)=t\left(g\left(j_{3}\right)=t\right.$, respectively).

(9) $f \circ F$ and $g$ are symmetric with respect to the points $r$ and $s$.

Then there exists a finite set $L=[1, l]$ and functions $G$ and $H$ of $L$ into $R$ and $Q$, 
respectively, such that

(10) $G(1)=H(1)=1, G(l)=r, H(l)=s$ and $f \circ F \circ G(m)=g \circ H(m)$ for every $m \in L$. 1].

(11) $|G(m+1)-G(m)| \leqslant 1$ and $|H(m+1)-H(m)|<1$ for every $m \in[1, l-$

Proof. See Figures 1-3. The proof will be by induction over $n$. Although the lemma is stated for functions $f, g$ and $F$ of segments of positive integers starting with 1 , we will, when using the induction step, consider functions of any segment of positive integers into $N$. We will only in Step 2, Case 1, indicate how one could (reversely if necessary) relabel the sets $P_{\alpha}, Q_{\alpha}$ and $R_{\alpha}$ such that one obtains functions of segments of positive integers starting with 1 . We will for each segment indicate which endpoint is considered to be the first element (i.e. corresponds to 1 after relabeling).

If $n=0$, the lemma is trivial. Assume the lemma is true for functions $f, g$ and $F$, and natural numbers $k, k<n-1$, satisfying (4)-(9), where $n$ is replaced by $k$. Let $P, Q, R, f, g, F$ and $s$ be as in Lemma 3.1 and let $\left(t_{0}, k_{0}\right)$ be the first element of the local maxima sequence of $f \circ F$.

If $g(j)>t_{0}$ for some $j \in(1, q)$, then it follows from the definition of $t_{0}$, (3)(iii), where $v=-1$, and (4) that $j>s-1$. Let $\hat{q}=\min \left\{j \in Q \mid g(j)>t_{0}\right\}$ and $\hat{g}=$ $g \mid[1, \hat{q}]$; then the functions $f, \hat{g}, F$ and the number $s \in \hat{Q}=[1, \hat{q}]$ satisfy (4)-(9). For convenience we will assume that $\hat{q}=q$ and $\hat{g}=g$. Similarly we may assume that $f(i)<t_{0}$ for each $i \in(1, p)$.

Let $x_{1}<x_{2}<\cdots<x_{a}, z_{1}<z_{2}<\cdots<z_{b}$ and $y_{1}<y_{2}<\cdots<y_{c}=j_{0}$ be all values of $R, P$ and $\left[1, j_{0}\right] \subset Q$, where $j_{0}$ is defined in (3), such that

$$
f \circ F\left(x_{i}\right)=f\left(z_{d}\right)=g\left(y_{j}\right)=t_{0} \quad(1<i<a, 1<d<b \text { and } 1<j<c) .
$$

Notice that $a, b \geqslant 1$ by the definition of the local maxima sequence of $f \circ F$ and $c>1$ by (9). Put $x_{0}=z_{0}=y_{0}=2, x_{a+1}=r$,

$$
z_{b+1}=\left\{\begin{array}{l}
p, \text { if } f(p)<t_{0} \\
p-1, \text { otherwise }
\end{array}\right.
$$

and

$$
y_{c+1}=\left\{\begin{array}{l}
q, \quad \text { if } g(j)<t_{0} \text { for each } j \in\left(j_{0}, q\right) \text { and } g(q)<t_{0}, \\
q-1, \quad \text { if } g(j)<t_{0} \text { for each } j \in\left(j_{0}, q\right) \text { and } g(q)>t_{0} \\
\min \left\{j>j_{0} \mid g(j)=t_{0}\right\}, \quad \text { otherwise. }
\end{array}\right.
$$

If $R_{\alpha}$ is any subsegment of $R$, then we denote by $F_{\alpha}, F \mid R_{\alpha}\left(f_{\alpha}\right.$ and $g_{\alpha}$ are defined similarly). Since by (4) (the proof is trivial if $p=2$ ), $f(2)<f(1)$ and $g(2)<g(1)$, it follows from (7) that $f(2)=g(2)$. We consider three cases as follows.

Case 1. $f(2)=g(2) \neq t_{0}$. Put $i_{0}=1$.

Case 2. $f(2)=g(2)=t_{0}$, and there exists $i_{0}$ such that $i_{0}=\min \{i \in$ $[1, a] \mid f \circ F\left(x_{i}\right)=t_{0}$ and $\left.F\left(x_{i}\right) \neq 2\right\}-1$.

Case 3. $f(2)=g(2)=t_{0}$, and $F\left(x_{i}\right)=2$ for each $i \in[1, a]$. Let $\eta=\max \{F(k) \mid k$ $\in R\}, i_{1}=\min \{k \in R \mid F(k)=\eta\}$ and $i_{0}=\max \left\{i \in\left[1, i_{1}\right] \mid F\left(x_{i}\right)=2\right\}$. 
We will construct the set $L$ and the functions $G$ and $H$ in six steps.

Step 1. Cases 1-3. Put $L_{0}=\{1\}, G_{0}(1)=1=H_{0}(1)$.

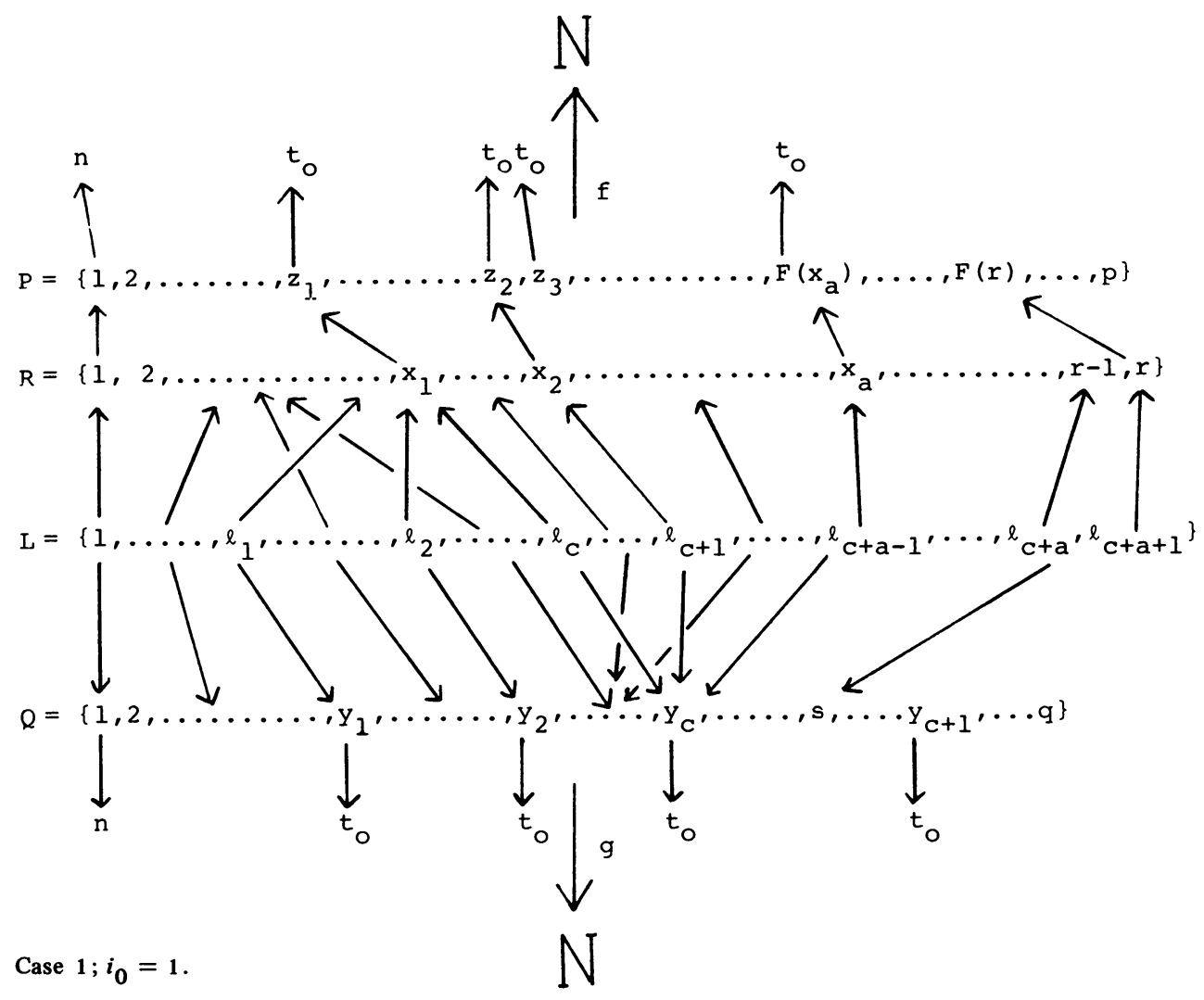

FIGURE 1

Step 2. Case 1. See Figures 1 and 2. Let $P_{1}=\left[2, z_{1}\right], Q_{1}=\left[2, y_{1}\right], R_{1}=\left[2, x_{1}\right]$ and $s_{1}=2$. We will reversely relabel the sets $P_{1}, Q_{1}$ and $R_{1}$. Let $p_{1}=z_{1}-1$, $q_{1}=y_{1}-1$ and $r_{1}=x_{1}-1$. Define functions $h_{1}: P_{1} \rightarrow P_{1}^{*}=\left[1, p_{1}\right], h_{2}: Q_{1} \rightarrow Q_{1}^{*}$ $=\left[1, q_{1}\right]$ and $h_{3}: R_{1} \rightarrow R_{1}^{*}=\left[1, r_{1}\right]$ by $h_{1}(i)=z_{1}+1-i, h_{2}(j)=y_{1}+1-j$ and $h_{3}(k)=x_{1}+1-k$. In this case we will say that $z_{1}, y_{1}$ and $r_{1}$ are the first elements of $P_{1}, Q_{1}$ and $R_{1}$, respectively.

We claim that the functions $f^{*}=f_{1} \circ h_{1}^{-1}, g^{*}=g_{1} \circ h_{2}^{-1}$, of $P_{1}^{*}$ and $Q_{1}^{*}$ into $N$ respectively and the function $F^{*}=h_{1} \circ F_{1} \circ h_{3}^{-1}: R_{1}^{*} \rightarrow P_{1}^{*}$ satisfy all conditions of Lemma 3.1, where $s^{*}=h_{2}\left(s_{1}\right)=h_{2}(2)=q_{1}$.

(4) $f^{*}(1)=f_{1} \circ h_{1}^{-1}(1)=f\left(z_{1}\right)=t_{0}<n-1$. Similarly, $g^{*}(1)=t_{0}$ and by the definition of $z_{1}, y_{1}$ and $t_{0}, f^{*}(i), g^{*}(j)<t_{0}-1$, if $i \in\left(1, p_{1}\right)$ and $j \in\left(1, q_{1}\right)$.

(5) $F^{*}(1)=h_{1} \circ F_{1} \circ h_{3}^{-1}(1)=h_{1} \circ F_{1}\left(x_{1}\right)=h_{1}\left(z_{1}\right)=1$ and $f^{*} \circ F^{*}\left(r_{1}\right)=$ $f_{1} \circ F_{1} \circ h_{3}^{-1}\left(r_{1}\right)=f_{1}(2)=g_{1}(2)=g^{*}\left(s^{*}\right)$. By the definition of $f^{*}, F^{*}, z_{1}$ and (4), we have $f^{*} \circ F^{*}(k)<t_{0}-1$ if $k \in\left(1, r_{1}\right)$. 


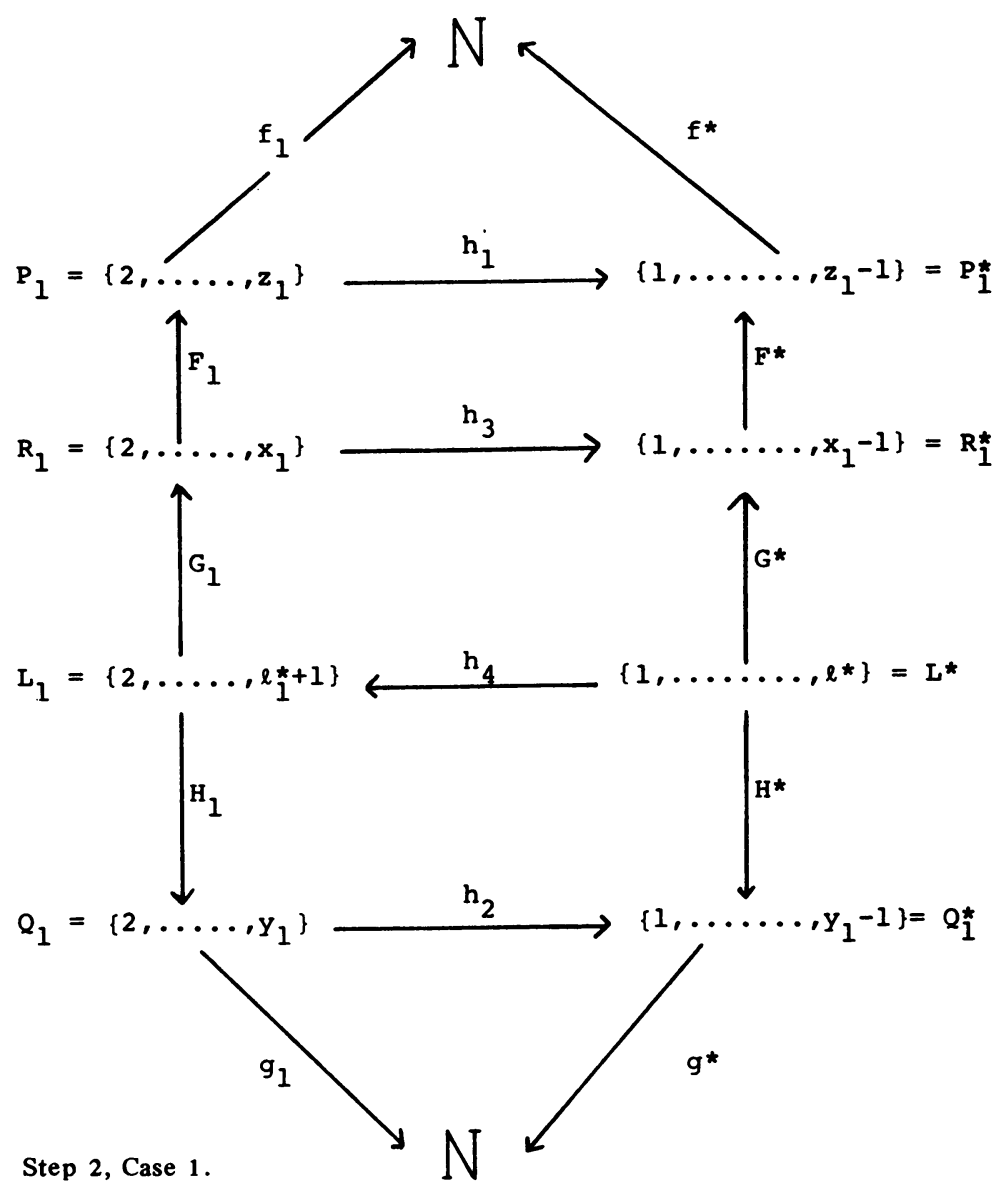

FIGURE 2

(6), follows from (6) and the definition of $F^{*}$.

$(7)_{1}$. By (7), the definitions of $f^{*}, g^{*}, z_{1}$ and $y_{1}$ we have that $f^{*}$ and $g^{*}$ are uniform of order $t_{0}$.

(8), follows from (8) and the definitions of $f^{*}$ and $g^{*}$.

(9). Let $\left\{\left(t_{v}^{\prime}, k_{v}^{\prime}\right)\right\}, v \in\left[0, \gamma_{1}\right]$, be the local maxima sequence of $f^{*} \circ F^{*}$ and put $t_{-1}^{\prime}=t_{0}, k_{-1}^{\prime}=1$. Since $f(2)=g(2)<f(1)=g(1)=n$, there exists, by (7), a number $k(n)>0$ and a sequence $n=a_{0}>a_{1}>\cdots>a_{k(n)}=0$ such that

(12) $f(1+u)=a_{u}=g(1+u)$ for $u \in[0, k(n)]$.

Hence by the definition of $f^{*}$ and $g^{*}$ we have

(13) $f^{*}\left(z_{1}-u\right)=a_{u}=g^{*}\left(y_{1}-u\right)$ for $u \in[1, k(n)]$.

Choose $v \in\left[-1, \gamma_{1}\right]$. We consider three cases as follows.

Case a. $0<v<\gamma_{1}$. Then $t_{v}^{\prime}>f^{*} \circ F^{*}\left(r_{1}\right)=f(2)=g(2)$. In particular, by the definition of $t_{0}^{\prime}, t_{0}>t_{0}^{\prime}>t_{v}^{\prime}>g(2)$. Hence by (12), there exists $j^{\prime} \in\left(1, y_{1}\right)$ such that $g\left(j^{\prime}\right)=0$. Since $g(1)=n, g\left(y_{1}\right)=t_{0}$ and $t_{v}^{\prime}<\min \left\{n, t_{0}\right\}$, it follows from (8) that there exist $j \in\left(1, y_{1}\right)$ such that $g(j)=t_{v}$, or equivalently, there exists $j \in Q_{1}^{*}$ such 
that $g^{*}(j)=t_{v}^{\prime}$. Let $j_{v}=\max \left\{j \in Q_{1}^{*} \mid g^{*}(j)=t_{v}^{\prime}\right\}$. Then $j_{v}<q_{1}-k(n)+1$ by the definition of $t_{v}^{\prime}$ and (13). We will verify (3) of the definition of symmetry.

(i) $g^{*}\left(j_{v}\right)=t_{v}^{\prime}$ by definition. Let $j \in\left(j_{v}, s^{*}\right)=\left(j_{v}, q_{1}\right)$ and suppose $g^{*}(j)>t_{v}^{\prime}$. Then $g^{*}(j)>t_{v}^{\prime}$ by the definition of $j_{v}$. Hence it follows as above from (8), that there exists $j^{\prime} \in\left(j_{v}, q_{1}\right)$ such that $g^{*}\left(j^{\prime}\right)=t_{v}^{\prime}$, contrary to the definition of $j_{v}$.

(ii) Let $k \in\left(k_{v}^{\prime}, r_{1}\right)$. By definition of $k_{v}^{\prime}$, we have $t=f^{*} \circ F^{*}(k)<t_{v}^{\prime}=$ $\min \left\{n, t_{v}^{\prime}\right\}$. If $F^{*}(k)=p_{1}-u$ for some $u \in[0, k(n)-1]$, put $j^{*}=q_{1}-u$. If $F^{*}(k) \neq p_{1}-u$ for each $u \in[0, k(n)-1]$, then it follows as above from (8), that there exists $j \in\left[j_{v}, q_{1}\right]$ such that $g^{*}(j)=t$. Let $j^{*}=\max \left\{j \in Q_{1}^{*} \mid g^{*}(j)=t\right\}$. Then $g^{*}\left(j^{*}\right)=t$ and $g^{*}(j)<t_{v}^{\prime}$ for each $j \in\left(j^{*}, q_{1}\right)=\left(j^{*}, s^{*}\right)$.

(iii) Let $j \in\left(j_{v}, s^{*}\right)=\left(j_{v}, q_{1}\right)$. Then by (i), $t=g^{*}(j)<t_{v}^{\prime}$. It follows as above, reversing the roles of $f$ and $g$, that there exists $i \in\left[F^{*}\left(k_{v}^{\prime}\right), p_{1}\right]$ such that $f^{*}(i)=t$. Hence by (6), there exists $k \in\left[k_{v}^{\prime}, r_{1}\right]$ such that $F^{*}(k)=i$ and whence $f^{*} \circ F^{*}(k)$ $=t$.

Case b. $v=-1$. Then $t_{-1}^{\prime}=t_{0}$ and $k_{-1}^{\prime}=1=j_{-1}$. We will verify (3).

(i) $g^{*}\left(j_{v}\right)=g^{*}(1)=t_{0}$ and by $(4)_{1}, g^{*}(j)<t_{0}$ if $j \in\left(1, q_{1}\right)=\left(j_{-1}, s^{*}\right)$.

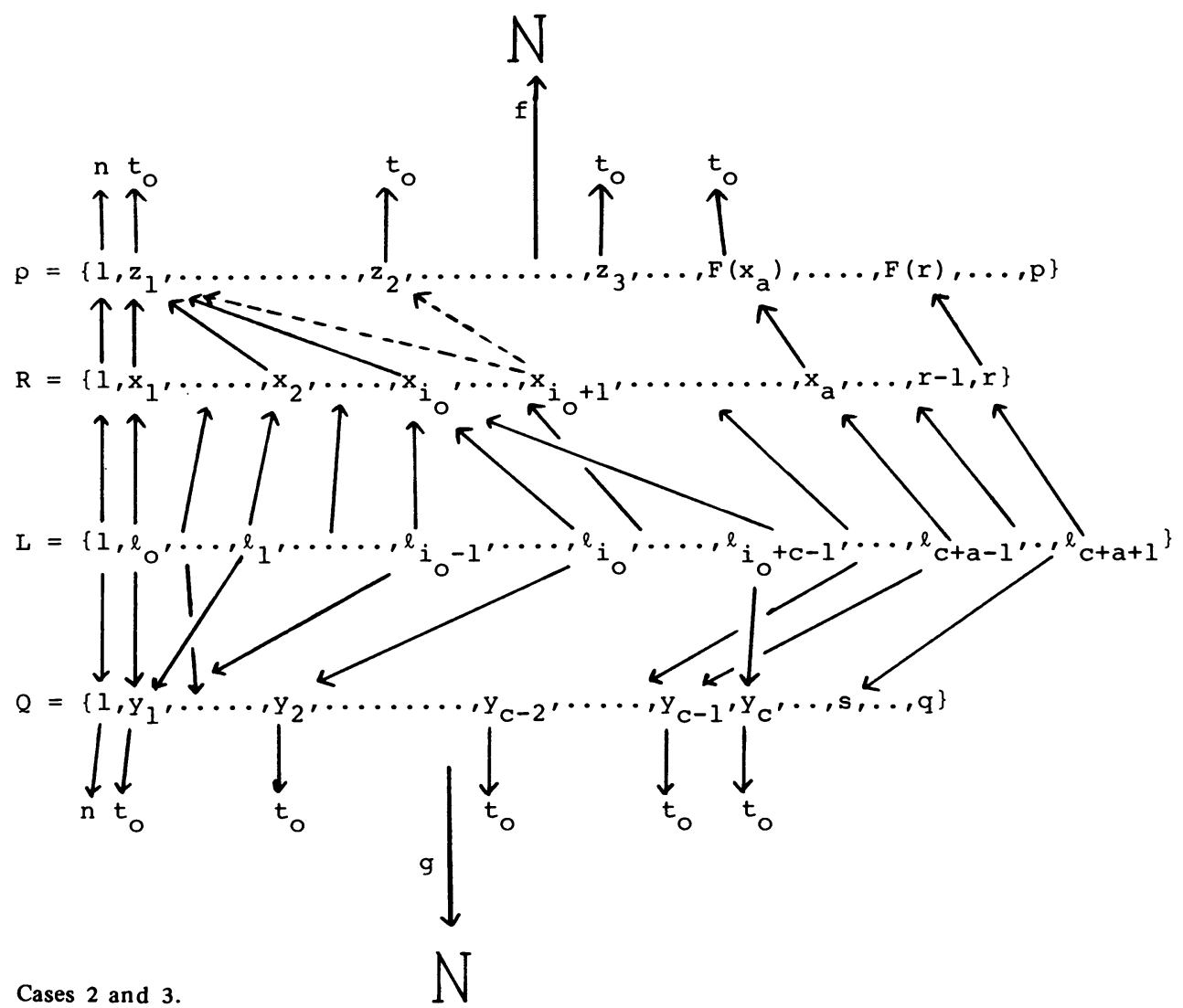

Figure 3 
(ii) Let $k \in\left(1, r_{1}\right)$, then by $(5)_{1}, f^{*} \circ F^{*}(k)<t_{0}$. Since there exists $j^{\prime} \in\left(1, y_{1}\right)$ (compare the proof of $\left.(4)_{1}\right)$ such that $g\left(j^{\prime}\right)=0$, we have by (8) that there exists $j^{\prime \prime} \in\left[2, y_{1}\right]$ such that $g\left(j^{\prime \prime}\right)=f^{*} \circ F^{*}(k)$, or, equivalently, there exists $j^{*} \in\left[1, q_{1}\right]$ such that $g^{*}\left(j^{*}\right)=f^{*} \circ F^{*}(k)$. By (4), we have $g^{*}(j)<t_{0}$ if $j \in\left(j^{*}, s^{*}\right)$.

(iii) Let $j \in\left(1, s^{*}\right)=\left(1, q_{1}\right)$. Then $g^{*}(j)<t_{0}$ and it follows as above from (8) and (6), that there exists $k \in\left[1, r_{1}\right]$ such that $f^{*} \circ F^{*}(k)=g^{*}(j)$.

Case c. $v=\gamma_{1}$. Then $f^{*} \circ F^{*}\left(r_{1}\right)=t_{v}^{\prime}=f^{*}\left(p_{1}\right)$ and $t_{v}^{\prime}=r_{1}$. Put $j_{v}=q_{1}=s^{*}$; then (i)-(iii) are automatically satisfied.

Hence in all cases, (i)-(iii) hold and we conclude that $f^{*} \circ F^{*}$ and $g^{*}$ are symmetric with respect to the points $r_{1}$ and $s^{*}$.

It follows from the induction hypothesis that there exists a set $L^{*}=\left[1, l^{*}\right]$ and functions $G^{*}$ and $H^{*}$ of $L^{*}$ into $R_{1}^{*}$ and $Q_{1}^{*}$, respectively, satisfying $(10)_{1}-(11)_{1}$. Let $l_{1}=l^{*}+1$ and define $h_{4}: L^{*} \rightarrow L_{1}=\left[1, l_{1}\right]$ by $h_{4}(m)=l^{*}+2-m$. Define functions $G_{1}: L_{1} \rightarrow R_{1}$ and $H_{1}: L_{1} \rightarrow Q_{1}$ by $G_{1}=h_{3}^{-1} \circ G_{1}^{*} \circ h_{4}^{-1}$ and $H_{1}=$ $h_{2}^{-1} \circ H_{1}^{*} \circ h_{4}^{-1}$, respectively. Then $G_{1}(2)=2=H_{1}(2), G_{1}\left(l_{1}\right)=x_{1}, H_{1}\left(l_{1}\right)=y_{1}$ and (10)-(11) are satisfied on $L_{1}$.

Cases 2 and 3. If $i_{0}>1$, then we define for each $\alpha \in\left[1, i_{0}-1\right]$ a set $L_{\alpha}$ and functions $G_{\alpha}$ and $H_{\alpha}$ of $L_{\alpha}$ into $R$ and $Q$ as follows. Let $P_{\alpha}=\left[z_{1}, z_{2}\right], R_{\alpha}=$ $\left[x_{\alpha}, x_{\alpha+1}\right]$ and $Q_{\alpha}=\left[y_{1}, y_{2}\right]$. One could relabel the sets $P_{\alpha}, Q_{\alpha}$ and $R_{\alpha}$ such that $z_{1}$, $y_{1}$ and $x_{\alpha}$ correspond to 1 after relabeling.

It follows that the functions $f_{\alpha}, g_{\alpha}$ and $F_{\alpha}$ satisfy $(4)_{\alpha}-(9)_{\alpha}$, where $z_{1}, y_{1}$ and $x_{\alpha}$ are the first elements of $P_{\alpha}, Q_{\alpha}$ and $R_{\alpha}$ respectively and $s_{\alpha}=2$. Hence there exists a set $L_{\alpha}=\left[l_{\alpha-1}, l_{\alpha}\right]\left(l_{\alpha-1}<l_{\alpha}\right)$ and functions $G_{\alpha}$ and $H_{\alpha}$ of $L_{\alpha}$ into $R_{\alpha}$ and $Q_{\alpha}$ respectively, satisfying $(10)_{\alpha}-(11)_{\alpha}$. In particular, $G_{\alpha}\left(l_{\alpha-1}\right)=x_{\alpha}, G_{\alpha}\left(l_{\alpha}\right)=x_{\alpha+1}$, and $H_{\alpha}\left(l_{\alpha-1}\right)=y_{1}=H_{\alpha}\left(l_{\alpha}\right)$.

Define $L_{i_{0}}=\left\{l_{i_{0}}\right\}\left(l_{i_{0}}=l_{i_{0}-1}+1\right)$ and functions $G_{i_{0}}$ and $H_{i_{0}}$ of $L_{i_{0}}$ into $R$ and $Q$ respectively, by $G_{i_{0}}\left(l_{i_{0}}\right)=x_{i_{0}}$ and $H_{i_{0}}\left(l_{i_{0}}\right)=y_{1}$.

Step 3. Case 1. Let $P_{\alpha}=\left[z_{0}, z_{1}\right], Q_{\alpha}=\left[y_{\alpha-i_{0}}, y_{\alpha+1-i_{0}}\right], R_{\alpha}=\left[x_{0}, x_{1}\right]$ and $R_{\alpha}^{*}=$ [4 $-x_{1}, x_{1}$ ]. Define a function $\Phi_{\alpha}: R_{\alpha}^{*} \rightarrow R_{\alpha}$ by

$$
\Phi_{\alpha}(k)=\left\{\begin{array}{l}
k, \quad \text { if } 2<k<x_{1}, \\
4-k, \text { if } 4-x_{1}<k<2,
\end{array}\right.
$$

and a function $F_{\alpha}^{*}: R_{\alpha}^{*} \rightarrow P_{\alpha}$ by $F_{\alpha}^{*}=F_{\alpha} \circ \Phi_{\alpha}\left(\alpha=i_{0}+1, \ldots, i_{0}+c-1\right)$ and consider two cases as follows.

Case a. $y_{\alpha-i_{0}}+1=y_{\alpha-i_{0}+1}$. Let $l_{\alpha}=l_{\alpha-1}+1, L_{\alpha}=\left\{l_{\alpha-1}, l_{\alpha}\right\}$ and define functions $G_{\alpha}$ and $H_{\alpha}$ of $L_{\alpha}$ into $R$ and $Q$, respectively, by $G_{\alpha}\left(l_{\alpha}\right)=G_{\alpha}\left(l_{\alpha-1}\right)=x_{1}$, $H_{\alpha}\left(l_{\alpha-1}\right)=y_{\alpha-i_{0}}$ and $H_{\alpha}\left(l_{\alpha}\right)=y_{\alpha-i_{0}+1}$.

Case b. $y_{\alpha-i_{0}}+1<y_{\alpha-i_{0}+1}$. Then the functions $f_{\alpha}, g_{\alpha}$ and $F_{\alpha}^{*}$ satisfy (4) $-(9)_{\alpha}$, where $z_{1}, y_{\alpha-i_{0}}$ and $4-x_{1}$ are the first elements of $P_{\alpha}, Q_{\alpha}$ and $R_{\alpha}^{*}$ respectively and $s_{\alpha}=y_{\alpha+1-i_{0}}$. It follows that there exists a set $L_{\alpha}$ and functions $G_{\alpha}$ and $H_{\alpha}$ of $L_{\alpha}$ into $R_{\alpha}^{*}$ and $Q_{\alpha}$, respectively, where $L_{\alpha}=\left[l_{\alpha-1}, l_{\alpha}\right]\left(l_{\alpha-1}<l_{\alpha}\right)$, satisfying $(10)_{\alpha^{-}}$ (11) $)_{\alpha}$. Define a function $G_{\alpha}: L_{\alpha} \rightarrow R_{\alpha}$ by $G_{\alpha}=\Phi_{\alpha} \circ G_{\alpha}^{*}$; then the functions $G_{\alpha}$ and $H_{\alpha}$ satisfy $(10)_{\alpha}-(11)_{\alpha}$. In particular, $G_{\alpha}\left(l_{\alpha-1}\right)=x_{1}=G_{\alpha}\left(l_{\alpha}\right), H_{\alpha}\left(l_{\alpha-1}\right)=y_{\alpha-i_{0}}$ and $H_{\alpha}\left(l_{\alpha}\right)=y_{\alpha+1-i_{0}}$. 
Cases 2 and 3. Let $P_{\alpha}=\left[z_{1}, z_{2}\right], Q_{\alpha}=\left[y_{\alpha-i_{0}}, y_{\alpha+1-i_{0}}\right], R_{\alpha}=\left[x_{i_{0}}, x_{i_{0}+1}\right]$ and $R_{\alpha}^{*}=\left[x_{i_{0}}, 2 x_{i_{0}+1}-x_{i_{0}}-2\right]$. Define a function $\Phi_{\alpha}: R_{\alpha}^{*} \rightarrow R_{\alpha}$ by

$$
\Phi_{\alpha}(k)=\left\{\begin{array}{l}
k, \text { if } x_{i_{0}}<k<x_{i_{0}+1}-1, \\
2 x_{i_{0}+1}-2-k, \text { if } x_{i_{0}+1}-1<k<2 x_{i_{0}+1}-x_{i_{0}}-2,
\end{array}\right.
$$

and a function $F_{\alpha}^{*}: R_{\alpha}^{*} \rightarrow R_{\alpha}$ by $F_{\alpha}^{*}=F_{\alpha} \circ \Phi_{\alpha}\left(\alpha=i_{0}+1, \ldots, i_{0}+c-1\right)$. Consider the following two cases.

Case a. $y_{\alpha-i_{0}}+1=y_{\alpha-i_{0}+1}$. Put $l_{\alpha}=l_{\alpha-1}+1, L_{\alpha}=\left\{l_{\alpha-1}, l_{\alpha}\right\}$ and define functions $G_{\alpha}$ and $H_{\alpha}$ of $L_{\alpha}$ into $R_{\alpha}$ and $Q_{\alpha}$, respectively, by $G_{\alpha}\left(l_{\alpha-1}\right)=G_{\alpha}\left(l_{\alpha}\right)=x_{i_{0}}$, $H_{\alpha}\left(l_{\alpha-1}\right)=y_{\alpha-i_{0}}$ and $H_{\alpha}\left(l_{\alpha}\right)=y_{\alpha-i_{0}+1}$.

Case b. $y_{\alpha-i_{0}}+1<y_{\alpha-i_{0}+1}$. Then the functions $f_{\alpha}, g_{\alpha}$ and $F_{\alpha}^{*}$ satisfy conditions $(4)_{\alpha}-(9)_{\alpha}$, where $z_{1}, y_{\alpha-i_{0}}$ and $x_{i_{0}}$ are the first elements of $P_{\alpha}, Q_{\alpha}$ and $R_{\alpha}^{*}$ respectively, and $s_{\alpha}=y_{\alpha+1-i_{0}}$. Hence there exists a set $L_{\alpha}=\left[l_{\alpha-1}, l_{\alpha}\right], l_{\alpha-1}<l_{\alpha}$, and functions $G_{\alpha}^{*}$ and $H_{\alpha}$ of $L_{\alpha}$ into $R_{\alpha}^{*}$ and $Q_{\alpha}$, respectively, satisfying $(10)_{\alpha}-(11)_{\alpha}$. Define a function $G_{\alpha}: L_{\alpha} \rightarrow R_{\alpha}$ by $G_{\alpha}=\Phi_{\alpha} \circ G_{\alpha}^{*}$. Then the functions $G_{\alpha}$ and $H_{\alpha}$ satisfy $(10)_{\alpha}-(11)_{\alpha}$. In particular, $G_{\alpha}\left(l_{\alpha-1}\right)=G_{\alpha}\left(l_{\alpha}\right)=x_{i_{0}}, H_{\alpha}\left(l_{\alpha-1}\right)=y_{\alpha-i_{0}}$ and $H_{\alpha}\left(l_{\alpha}\right)=y_{\alpha+1-i_{0}}$.

Step 4. We will, in Step 4, consider three subcases of Cases 1, 2 and 3.

Case a. $y_{c-1}<y_{c}-1$. Let $R_{\alpha}=\left[x_{\alpha-c}, x_{\alpha-c+1}\right], z_{i(\alpha)}=F\left(x_{\alpha-c}\right)$,

$$
z_{j(\alpha)}= \begin{cases}z_{i(\alpha)-1}, & \text { if } F(x)<z_{i(\alpha)} \text { for some } x \in R_{\alpha}, \\ z_{i(\alpha)+1}, & \text { otherwise, }\end{cases}
$$

$P_{\alpha}=\left[z_{i(\alpha)}, z_{j(\alpha)}\right]$ and $Q_{\alpha}=\left[y_{c-1}, y_{c}\right],\left(\alpha=c+i_{0}, \ldots, c+a-1\right)$.

If $\left|z_{i(\alpha)}-z_{j(\alpha)}\right|<1$, then by (6) and the definition of $\left\{x_{i}\right\}(i=1,2, \ldots, a)$ and $z_{j(\alpha)}$, we have $x_{\alpha-c}+1=x_{\alpha-c+1}$. Put $l_{\alpha}=l_{\alpha-1}+1, L_{\alpha}=\left[l_{\alpha-1}, l_{\alpha}\right]$ and define functions $G_{\alpha}$ and $H_{\alpha}$ of $L_{\alpha}$ into $R$ and $Q$, respectively by $G_{\alpha}\left(l_{\alpha-1}\right)=x_{\alpha-c}$, $G_{\alpha}\left(l_{\alpha}\right)=x_{\alpha-c+1}$ and $H_{\alpha}\left(l_{\alpha-1}\right)=y_{c}=H\left(l_{\alpha}\right)$.

If $\left|z_{i(\alpha)}-z_{j(\alpha)}\right|>1$, then the functions $f_{\alpha}, g_{\alpha}$ and $F_{\alpha}$ satisfy conditions (4) $\alpha-(9)_{\alpha}$, where $x_{\alpha-c}, z_{i(\alpha)}$ and $y_{c}$ are the first elements of $R_{\alpha}, P_{\alpha}$ and $Q_{\alpha}$ respectively and $s_{\alpha}=y_{c}$. Hence there exists a set $L_{\alpha}=\left[l_{\alpha-1}, l_{\alpha}\right], l_{\alpha-1}<l_{\alpha}$, and functions $G_{\alpha}$ and $H_{\alpha}$ of $L_{\alpha}$ into $R_{\alpha}$ and $Q_{\alpha}$ respectively, satisfying $(10)_{\alpha}-(11)_{\alpha}$. In particular, $G_{\alpha}\left(l_{\alpha-1}\right)=$ $x_{\alpha-c}, G_{\alpha}\left(l_{\alpha}\right)=x_{\alpha-c+1}$ and $H_{\alpha}\left(l_{\alpha-1}\right)=H_{\alpha}\left(l_{\alpha}\right)=y_{c}$.

Case b. $y_{c-1}=y_{c}-1$ and there exists $k \in\left[x_{i_{0}}, r\right]$ such that $f \circ F(k) \neq g(j)$ for each $j \in\left[y_{c}, y_{c+1}\right]$. Define $R_{\alpha}$ and $P_{\alpha}$ as above. Put $s_{\alpha}=y_{c-1}$ and $Q_{\alpha}=$ $\left[y_{c-2}, y_{c-1}\right]$. It follows as above that there exists a set $L_{\alpha}=\left[l_{\alpha-1}, l_{\alpha}\right], l_{\alpha-1}<l_{\alpha}$, and functions $G_{\alpha}$ and $H_{\alpha}$ of $L_{\alpha}$ into $R_{\alpha}$ and $Q_{\alpha}$, respectively satisfying $(10)_{\alpha}-(11)_{\alpha}$ and such that $G_{\alpha}\left(l_{\alpha-1}\right)=x_{\alpha-c}, G_{\alpha}\left(l_{\alpha}\right)=x_{\alpha-c+1}$ and $H_{\alpha}\left(l_{\alpha-1}\right)=y_{c}=H_{\alpha}\left(l_{\alpha}\right)$.

Case c. $y_{c-1}=y_{c}-1$ and if $k \in\left[x_{i_{0}}, r\right]$, then $f \circ F(k)=g(j)$ for some $j \in$ $\left[y_{c}, y_{c+1}\right]$. Define $R_{\alpha}, P_{\alpha}, f_{\alpha}, F_{\alpha}$ as above. Let $s_{\alpha}=y_{c}, Q_{\alpha}=\left[y_{c}, y_{c+1}\right]$. It follows as above that there exists a set $L_{\alpha}$ and functions $G_{\alpha}$ and $H_{\alpha}$ of $L_{\alpha}$ into $R$ and $Q$ respectively satisfying $(10)_{\alpha}-(11)_{\alpha}$.

Step 5. Cases 1, 2 and 3. If $r-x_{a}<1$, put $l_{\alpha}=l_{\alpha-1}+1, L_{\alpha}=\left\{l_{\alpha}\right\}$, and define functions $G_{\alpha}$ and $H_{\alpha}$ of $L_{\alpha}$ into $R$ and $Q$ by $G_{\alpha}\left(l_{\alpha}\right)=G_{\alpha-1}\left(l_{\alpha-1}\right)$ and $H_{\alpha}\left(l_{\alpha}\right)=$ $H_{\alpha-1}\left(l_{\alpha-1}\right)$, respectively. If $r-x_{a}>1$, let $R_{\alpha}=\left[x_{a}, r-1\right], F\left(x_{a}\right)=z_{i(\alpha)}$, 


$$
z_{j(\alpha)}= \begin{cases}z_{i(\alpha)+1}, & \text { if } F(r)>z_{i(\alpha)}, \\ z_{i(\alpha)-1}, & \text { if } F(r)<z_{i(\alpha)},\end{cases}
$$

and

$$
y_{c}^{*}= \begin{cases}y_{c+1}, & \text { if } s>y_{c}, \\ y_{c-1}, & \text { if } s<y_{c} .\end{cases}
$$

Then the functions $f_{\alpha}, g_{\alpha}$ and $F_{\alpha}$ satisfy (4) $-(9)_{\alpha}$ (in this case (9) $)_{\alpha}$ follows from (9)), where $x_{a}, y_{c}$ and $z_{i(\alpha)}$ are the first elements of $R_{\alpha}, Q_{\alpha}$ and $P_{\alpha}(\alpha=c+a)$.

Hence in both cases, there exists a set $L_{\alpha}$ and functions $G_{\alpha}$ and $H_{\alpha}$ of $L_{\alpha}$ into $R_{\alpha}$ and $Q_{\alpha}$ satisfying $(10)_{\alpha}-(11)_{\alpha}$.

Step 6. Cases 1, 2 and 3. Put $l_{\alpha}=l_{\alpha-1}+1, L_{\alpha}=\left\{l_{\alpha}\right\}$. Define functions $G_{\alpha}$ and $H_{\alpha}$ of $L_{\alpha}$ into $R$ and $Q$ by $G_{\alpha}\left(l_{\alpha}\right)=r$ and $H_{\alpha}\left(l_{\alpha}\right)=s$, respectively $(\alpha=c+a+1)$.

Let $L=L_{0} \cup L_{1} \cup \cdots \cup L_{c+a+1}$. Define functions $G: L \rightarrow R$ and $H: L \rightarrow Q$ by $G(m)=G_{\alpha}(m)$ and $H(m)=H_{\alpha}(m)$, respectively, if $m \in L_{\alpha}$. It follows from the definitions of $G$ and $H$ that $G$ and $H$ satisfy conditions (10)-(11) and the proof is complete.

4. Tracing continua in the unit square. Let $C$ be a subcontinuum of $I^{2}$ and $\left\{\left(x_{i}, 0\right)\right\}(i=1,2, \ldots, n)$ a sequence of points in $I^{2}$, where $0=x_{1}<x_{2}<\cdots<$ $x_{n}=1$. If $(a, t)$ and $\left(b, t^{*}\right)$ are two points of $C$ and $\eta>0$, then we say that $(a, t)$ and $\left(b, t^{*}\right)$ can be joined by a finite chain, provided there exists a finite sequence $C_{1}, C_{2}, \ldots, C_{p}$ of subcontinua of $C$ such that the following three conditions are satisfied.

(1) $(a, t) \in C_{1}$ and $\left(b, t^{*}\right) \in C_{p}$.

(2) For each $j, 1<j<p$, there exists an $i \in[1, n-1]$ such that $C_{j} \subset\left[x_{i}, x_{i+1}\right]$ $\times I$.

(3) For each $j, 1<j<p$, there exists an $s \in[1, n]$ such that the following two conditions are satisfied.

(i) $C_{j} \cap\left[\left\{x_{s}\right\} \times I\right] \neq \varnothing \neq C_{j+1} \cap\left[\left\{x_{s}\right\} \times I\right]$,

(ii) $d\left(C_{j} \cap\left[\left\{x_{s}\right\} \times I\right], C_{j+1} \cap\left[\left\{x_{s}\right\} \times I\right]\right)<\eta$, where $d(\cdot, \cdot)$ denotes the regular metric of $I^{2}$.

4.1. Lemma. Let $C$ be a continuum in $I^{2}, \eta>0$, and $\left\{\left(x_{i}, 0\right)\right\}(i=1,2, \ldots, n) a$ sequence of points in $I^{2}$ such that

(4) $0=x_{1}<x_{2}<\cdots<x_{n}=1$,

(5) $C \cap\left(\left\{x_{1}\right\} \times I\right) \neq \varnothing$.

Then every two points of $C$ can be joined by a finite chain (relative to $\eta$ ).

Proof. Let $\left(a, t^{*}\right) \in C \cap\left(\left\{x_{1}\right\} \times I\right)$. It is sufficient to show that each point of $C$ can be joined by a finite chain to the point $\left(a, t^{*}\right)$. Let

$A=\left\{(y, t) \in C \mid\left(a, t^{*}\right)\right.$ and $(y, t)$ can be joined by a finite chain relative to $\left.\eta\right\}$.

Then $A \neq \varnothing$. We will show that $A$ is both open and closed in $C$.

Let $\left\{\left(y_{j}, t_{j}\right)\right\}(j=1,2, \ldots)$ be a sequence of points in $C$ converging to the point $\left(y_{0}, t_{0}\right) \in C$. If $y_{j} \in\left[x_{i}, x_{i+1}\right)$ for some $i \in[1, n-1]$, then we denote by $K_{j}$ the component of $C \cap\left(\left[x_{i}, x_{i+1}\right] \times I\right)$ containing the point $\left(y_{j}, t_{j}\right)$ and if $y_{j}=1$, then 
$K_{j}$ denotes the component of $C \cap\left(\left[x_{n-1}, x_{n}\right] \times I\right)$ containing the point $\left(y_{j}, t_{j}\right)$ $(j=0,1,2, \ldots)$. It follows [7, p. 171] that $\mathrm{Ls} K_{j}$ is a subcontinuum of $C$ and we may assume that $\operatorname{Ls} K_{j} \subset\left[x_{i}, x_{i+1}\right] \times I$ for some $i \in[1, n-1]$. By (5) and the fact that $C$ is a continuum, there exists a sequence of points, $\left\{\left(z_{j}, t_{j}^{*}\right)\right\},\left(z_{j}, t_{j}^{*}\right) \in K_{j}$ $(j=1,2, \ldots)$, such that $z_{j} \in\left\{x_{i}, x_{i+1}\right\}(j=1,2, \ldots)$ and we may assume that either $z_{j}=x_{i}$ or $z_{j}=x_{i+1}$ for each $j=1,2, \ldots$, and that the sequence $\left\{\left(z_{j}, t_{j}^{*}\right)\right\}$ converges to the point $\left(z_{0}, t_{0}^{*}\right) \in \operatorname{Ls} K_{j}$. Let $k>0$ such that $d\left(\left(z_{k}, t_{k}^{*}\right),\left(z_{0}, t_{0}^{*}\right)\right)<\eta$. If $C_{1}, C_{2}, \ldots, C_{p}$ is a chain joining $\left(a, t^{*}\right)$ and $\left(y_{j}, t_{j}\right)$, then we may assume

(6) $C_{p}=K_{j}$ if $j>0$ and $C_{p} \supset \operatorname{Ls} K_{j}$ if $j=0$.

$A$ is open. Suppose $A$ is not open in $C$. Then there exists a sequence of points $\left\{\left(y_{j}, t_{j}\right)\right\}$ in $C$ converging to a point $\left(y_{0}, t_{0}\right)$ such that $\left(y_{j}, t_{j}\right) \notin C(j=1,2, \ldots)$ and $\left(y_{0}, t_{0}\right) \in C$. Let $k$ and $K_{j}$ be defined as above, and let $C_{1}, C_{2}, \ldots, C_{p}$ be a finite chain joining $\left(a, t^{*}\right)$ and $\left(y_{0}, t_{0}\right)$, where $C_{p} \supset$ Ls $K_{j}$. Then $C_{1}, C_{2}, \ldots, C_{p}, K_{k}$ is a finite chain joining $\left(a, t^{*}\right)$ and $\left(y_{k}, t_{k}\right)$ relative to $\eta$, contrary to the assumption.

$A$ is closed. Suppose $\left\{\left(y_{j}, t_{j}\right)\right\}$ is a sequence of points in $A$ converging to the point $\left(y_{0}, t_{0}\right)$. Let $C_{1}, C_{2}, \ldots, C_{p}$ be a finite chain joining $\left(a, t^{*}\right)$ and $\left(y_{k}, t_{k}\right)$, where $C_{p}=K_{k}$. Then $C_{1}, C_{2}, \ldots, C_{p}$, Ls $K_{j}$ is a finite chain joining the points $\left(a, t^{*}\right)$ and $\left(y_{0}, t_{0}\right)$.

Hence $A$ is both open and closed in $C$ and the proof is complete.

4.2. Corollary. Let $C, I^{2}, \eta,\left\{\left(x_{i}, 0\right)\right\}(i=1,2, \ldots, n)$, be as in Lemma 4.1 and let $\left(a, t_{1}^{*}\right)$ and $\left(b, t_{2}^{*}\right)$ be two points of $C$ such that

(7) $\left|x_{i}-x_{i+1}\right|<\eta, i=1,2, \ldots, n-1$,

(8) $a=x_{c}$ and $b=x_{d}$ for some $c, d \in[1, n]$.

Then there exist a finite set $R=[1, r]$, a function $F: R \rightarrow[1, n]$, and a sequence $\left\{t_{k}\right\}$, $k=1,2, \ldots, r$, such that

(9) $F(1)=c, t_{1}=t_{1}^{*}, F(r)=d, t_{r}=t_{2}^{*}$ and $\left(x_{F(k)}, t_{k}\right) \in C$ for each $k \in[1, r]$,

(10) $|F(k+1)-F(k)|<1$, for each $k \in[1, r-1]$,

(11) for each $k \in[1, r-1]$ at least one of the following two conditions are satisfied.

(i) $F(k)=F(k+1)$ and $\left|t_{k}-t_{k+1}\right|<\eta$,

(ii) there exists a subcontinuum $C_{k}$ of $C$ such that $d\left(C_{k},\left(x_{F(k)}, t\right)\right)<\eta$ for each $t \in\left[t_{k}, t_{k+1}\right]$.

Proof. By Lemma 4.1 there exists a finite chain joining the points $\left(a, t_{1}^{*}\right)$ and $\left(b, t_{2}^{*}\right)$ relative to $\eta$. By (3), there exist for each $j \in[1, p)$ a pair of points $\left(x_{f(j)}, u_{j}\right) \in C_{j}$ and $\left(x_{f(j)}, v_{j}\right) \in C_{j+1}$ such that $\left|u_{j}-v_{j}\right|<\eta$. Let $r=2 p, R=[1, r]$, and define a function $F: R \rightarrow[1, n]$ and a sequence $\left\{t_{k}\right\}(k=1,2, \ldots, r)$ by

$$
F(k)= \begin{cases}c, & \text { if } k=1, \\ f(j), & \text { if } k=2 j, 2 j+1,1<j<p-1, \\ d, & \text { if } k=2 p\end{cases}
$$

and 


$$
t_{k}= \begin{cases}t_{1}^{*}, & \text { if } k=1, \\ u_{j}, & \text { if } k=2 j, 1<j<p-1, \\ v_{j}, & \text { if } k=2 j+1,1<j<p-1, \\ t_{2}^{*}, & \text { if } k=2 p\end{cases}
$$

By (2), $|F(k)-F(k+1)|<1$ for each $k \in[1, r-1]$. If $k=2 j$, then $F(k)=$ $F(k+1)$ and $\left|t_{k}-t_{k+1}\right|=\left|u_{j}-v_{j}\right|<\eta$, and if $F(k) \neq F(k+1)$, then $k=2 j+1$ for some $j \in[1, p-1]$ and hence $\left(x_{F(k)}, t_{k}\right),\left(x_{F(k+1)}, t_{k+1}\right) \in C_{j+1}$ and (11)(ii) follows from (2) and (7) and the proof is complete.

5. Four lemmas. The following lemmas are needed in the proof of Theorem 6.1.

5.1. LemMa. Let $X$ be a fan with vertex $v$, and $z$ and $y$ two points of $X$. Suppose there exist two sequences of points $\{y(i, 1)\}$ and $\{y(i, 2)\}(i=1,2, \ldots)$, in $X$, both converging to $y$ such that

(1) $z<y$ and $y(i, 1)<y(i, 2)(i=1,2, \ldots)$,

(2) $\operatorname{Lim}[y(i, 1), y(i, 2)]=[z, y]$,

(3) $v \in \operatorname{Ls}\{x \in X \mid x \geqslant y(i, 2)\}$.

Then $X$ is not contractible.

Proof. We will show that $X$ is of type $N$ (see $\$ 2$ ). We may assume that $v \in \operatorname{Lim}\{x \in X \mid x \geqslant y(i, 2)\}$. Choose sequences $\{z(i, 1)\},\{z(i, 2)\}$ and $\{z(i, 3)\}$ $(i=1,2, \ldots)$ such that

(4) $z=\lim z(i, 1)=\lim z(i, 2)=\lim z(i, 3)$,

(5) $z(i, 2)<y(i, 1)<z(i, 1)<y(i, 2)<z(i, 3)(i=1,2, \ldots)$.

Moreover we may assume (see [6, p. 5])

(6) $\operatorname{Ls}[z(i, 2), y(i, 1)] \geqslant z$ and $\operatorname{Ls}[y(i, 2), z(i, 3)]>z$.

Furthermore we may assume that

$$
\operatorname{Ls}[z(i, 2), y(i, 1)]=\operatorname{Lim}[z(i, 2), y(i, 1)]=[z, r]
$$

and

$$
\operatorname{Ls}[y(i, 2), z(i, 3)]=\operatorname{Lim}[y(i, 2), z(i, 3)]=[z, s]
$$

for some $r, s \in X$. We will consider the following two cases.

Case 1. $r<s$. Choose a sequence $\{r(i, 1)\}$, where $r(i, 1) \in[z(i, 2), y(i, 1)]$ $(i=1,2, \ldots)$, and using $[6$, p. 5], a sequence $\{r(i, 2)\}$, where $r(i, 2) \in[z(i, 3)$, $y(i, 2)](i=1,2, \ldots)$ such that

(7) $r=\lim r(i, 1)=\lim r(i, 2)$,

(8) $\operatorname{Ls}[y(i, 2), r(i, 2)]<r$,

where we take a subsequence and relabel if necessary.

Let $A_{i}=[z(i, 2), z(i, 1)]$ and $B_{i}=[r(i, 1), r(i, 2)]$. Then by (2), (4), (6) and (7), $\operatorname{Lim} A_{i}=[z, r]$ and by (2), (4), (7) and (8), Lim $B_{i}=[z, r]$. Hence $X$ is of type $N$ between the points $z$ and $r$.

Case 2. $r>s$. One can show, using a similar argument as above, that $X$ is of type $N$ between the points $z$ and $s$. The proof of this statement, being a replica of Case 1 , is omitted. 
In both cases $X$ is of type $N$ and hence by Theorem 2.1, $X$ is not contractible. This completes the proof of Lemma 5.1.

5.2. Remarks. It will follow from Theorem 6.1 that every contractible fan is locally connected at its vertex. Hence the conditions in 5.3-5.5 are never fulfilled (cf. Theorem 2.2).

5.3. LEMMA. Let $X$ be a contractible fan, where $H$ is a contraction of $X$, and $\left\{v_{i}\right\} a$ sequence of points in $X$ converging to the vertex $v$ of $X$ such that $\operatorname{Ls}\left[v, v_{i}\right]=K \neq$ $\{v\}$; then $K=H\left(\{v\} \times I_{0}\right)$ for some subset $I_{0}$ of $I$.

Proof. Let $H: X \times I \rightarrow X$ be the contraction of $X$ and let $t(i, 2)=\inf \{t \in$ $\left.I \mid H\left(v_{i}, t\right)=v\right\}$. Notice that $t(i, 2)$ exist for all except possibly one $i$ since $X$ is contractible and hereditarily unicoherent. Let

$$
t(i, 1)=\sup \left\{t \in[0, t(i, 2)] \mid H\left(v_{i}, t\right)=v_{i}\right\},
$$

and $I_{i}=[t(i, 1), t(i, 2)]$. Then $H\left(\left\{v_{i}\right\} \times I_{i}\right)=\left[v, v_{i}\right]$. Hence $K=\operatorname{Ls}\left[v, v_{i}\right]=$ Ls $H\left(\left\{v_{i}\right\} \times I_{i}\right)=H\left(\operatorname{Ls}\left\{v_{i}\right\} \times I_{i}\right)=H\left(\{v\} \times I_{0}\right)$ for some $I_{0} \subset I$.

5.4. Corollary. Let $X$ and $K$ be defined as in Lemma 5.3. Then $K$ is a dendrite.

Proof. By Lemma 5.3, $K=H\left(\{v\} \times I_{0}\right) \subset H(\{v\} \times I)$. Hence $K$, being a subcontinuum of a hereditarily unicoherent locally connected continuum, is locally connected.

Corollary 5.4 follows also from a stronger result, recently obtained by J. J. Charatonik and Z. Grabowski (see [5, Lemma 15, p. 235]).

5.5. LEMMA. Let $X$ be a contractible fan, $\alpha>0$, and $\left\{v_{i}\right\}$ a sequence of points in $X$ converging to the vertex $v$ of $X$ such that $K=\operatorname{Lim}\left[v, v_{i}\right] \neq\{v\}$. Suppose $x$ is a point of $K \backslash B(v, 3 \alpha)$, and let $U$ and $V$ be neighborhoods of $x$ and $v$ respectively such that

(9) $U \subset B\left(x, \frac{1}{2} \alpha\right)$ and $U \cap K$ is connected,

(10) $V \subset B(v, \alpha)$.

Then there exists an index $N>0$ such that if $i>N$, then for each component $C$ of $\left[v, v_{i}\right] \backslash V$, at most two components of $C \cap B(x, \alpha)$ intersect $\bar{U}$.

Proof. Suppose there exists a sequence of subscripts $i_{1}<i_{2}<i_{3}<\cdots$ and components $C\left(i_{n}\right)$ of $\left[v, v_{i_{n}} \backslash V\right.$ such that at least three components of $C\left(i_{n}\right) \cap$ $B(x, \alpha)$ intersect $\bar{U}(n=1,2, \ldots)$. Choose $a_{n}<b_{n}<c_{n}$ in three different components of $C\left(i_{n}\right) \cap B(x, \alpha)$ such that $a_{n}, b_{n}, c_{n} \in \bar{U}(n=1,2, \ldots)$. We may assume that the sequences $\left\{a_{n}\right\},\left\{b_{n}\right\}$ and $\left\{c_{n}\right\}$ converge to $a_{0}, b_{0}$ and $c_{0}$, respectively. Suppose

$$
X=\bigcup_{r \in R}\left\{J_{r} \mid J_{r} \simeq[0,1] \text { for each } r \in R \text { and } J_{r_{1}} \cap J_{r_{2}}=\{v\} \text { if } r_{1} \neq r_{2} \in R\right\},
$$

and $x \in J_{r_{0}}$. Let $\operatorname{Ls}\left[a_{n}, b_{n}\right] \cup \operatorname{Ls}\left[b_{n}, c_{n}\right]=H$. Then $H$ is a subcontinuum of $K \backslash\{v\}$. Hence by (9), $H \subset J_{r_{0}}$. We may assume that $\operatorname{Lim}\left[a_{n}, b_{n}\right]=[z, p]$ and $\operatorname{Lim}\left[b_{n}, c_{n}\right]=$ $[r, s]$ for some $z, p, r, s \in J_{r_{0}}$, where $z<p$ and $r<s$. Clearly either $z$ or $p$ (or both) belong to $K \backslash B(x, \alpha)$ and we consider two cases as follows. 
Case 1. $z \in K \backslash B(x, \alpha)$. Let $\left\{z_{n}\right\}, z_{n} \in\left[a_{n}, b_{n}\right](n=1,2, \ldots)$, be a sequence of points converging to $z$. Since $z \in J_{r_{0}} \backslash B(x, \alpha)$ and $[z, p] \cap \bar{U} \neq \varnothing$, we have $z<$ $\inf \left\{w \mid w \in \bar{U} \cap J_{r_{0}}\right\}=y$. Also, since $a_{0}, b_{0} \in \bar{U} \cap J_{r_{0}}$, we have $a_{0}, b_{0}>y$. Hence we may assume that there exist sequences $\{y(n, 1)\}$ and $\{y(n, 2)\}$ such that

(11) $y(n, 1) \in\left[a_{n}, z_{n}\right], y(n, 2) \in\left[z_{n}, b_{n}\right]$,

(12) $\lim y(n, 1)=\lim y(n, 2)=y$,

(13) $\operatorname{Ls}\left[y(n, 1), z_{n}\right]<y$ and $\operatorname{Ls}\left[y(n, 2), z_{n}\right]<y$,

where we take a subsequence and relabel if necessary. It follows that $\operatorname{Lim}[y(n, 1)$, $y(n, 2)]=[z, y]$, where $z<y$. Hence $X$ is not contractible by Lemma 5.1, contrary to the assumptions.

Case 2. $z \in B(x, \alpha)$. Then $p \in J_{r_{0}} \backslash B(x, \alpha)$. If $r \in K \backslash B(x, \alpha)$, then we conclude as in Case 1, replacing $a_{n}, b_{n}, z$ and $p$ by $b_{n}, c_{n}, r$ and $s$, respectively, that $X$ is not contractible. Hence we may assume that $r \in B(x, \alpha)$ and $s \in J_{r_{0}} \backslash B(x, \alpha)$.

Let $g=\inf \left\{w \mid w \in J_{r_{0}} \backslash B(x, \alpha)\right.$ and $\left.w>x\right\}$. By [6, p. 5], there exist sequences $\{g(n, 1)\}$ and $\{g(n, 2)\}$ such that both sequences converge to $g$ and

(14) $\operatorname{Ls}\left[b_{n}, g(n, 1)\right]<g, \operatorname{Ls}\left[b_{n}, g(n, 2)\right]<g$.

We may assume that $\operatorname{Lim}[g(n, 1), g(n, 2)]=[m, g]$ for some $m$. Hence $m<b<g$ and by Lemma $5.1, X$ is not contractible.

Hence in both cases we conclude that $X$ is not contractible, contrary to the assumptions, and the proof is complete.

Let $X$ be a fan with vertex $v$ and let $z_{1}$ and $z_{2}$ be two points of $X$, where $z_{1}<z_{2}$, and $\varepsilon>0$ such that

(15) $\left[z_{1}, z_{2}\right] \backslash B\left(z_{1}, 3 \varepsilon\right) \neq \varnothing, z_{2} \in B\left(z_{1}, \varepsilon\right)$ and $v \notin B\left(z_{1}, 3 \varepsilon\right)$.

Then we say that $z_{1}$ and $z_{2}$ are $\varepsilon$-related.

Suppose $z_{1}$ and $z_{2}$ are $\varepsilon$-related. Let

$$
d=\left\{\begin{array}{l}
\sup \left\{x \in X \mid x>z_{2}\right\}, \quad \text { if }\left\{x \in X \mid x>z_{2}\right\} \cap \operatorname{Bd}\left(B\left(z_{1}, 3 \varepsilon\right)\right)=\varnothing, \\
\inf \left\{x \in \operatorname{Bd}\left(B\left(z_{1}, 3 \varepsilon\right)\right) \mid x>z_{2}\right\}, \text { otherwise, }
\end{array}\right.
$$

and $c=\sup \left\{x \in \operatorname{Bd}\left(B\left(z_{1}, 3 \varepsilon\right)\right) \mid x<z_{2}\right\}$. Let $W=\left\{x \in B\left(z_{1}, \varepsilon\right) \mid c<x<d\right\}$. Then we say that $W$ is the $\varepsilon$-relative neighborhood of $z_{2}$ with respect to $z_{1}$.

5.6. Lemma. Let $X$ be a contractible fan, where $H: X \times I \rightarrow X$ is a contraction, and $\varepsilon, \eta>0$. Suppose $z_{1}$ and $z_{2}$ are two points of $X$ such that

(16) $\eta$ is the uniform continuity measure of $H$ for $\varepsilon$,

(17) $z_{1}$ and $z_{2}$ are $\varepsilon$-related.

Let $C$ be a component of $H^{-1}\left(z_{1}\right), W$ the e-relative neighborhood of $z_{2}$ with respect to $z_{1}$, and $F, G$, functions of a finite set $L=\{1,2, \ldots, l\}$ into $X$ such that

(18) $d(F(j), G(j))<\eta / 2, j \in[1, l]$,

(19) $\operatorname{diam}([G(j), G(j+1)])<\eta / 2, j \in[1, l-1]$,

(20) there is a sequence $\left\{t_{j}\right\}(j=1,2, \ldots, l)$ in I such that (i) $\left(F(j), t_{j}\right) \in C$,

$j \in[1, l]$,

and at least one of the following two conditions is satisfied for each $j \in[1, l-1]$

(ii) $F(j)=F(j+1)$ and $\left|t_{j}-t_{j+1}\right|<\eta / 2$,

(iii) $d(C,(F(j), t))<\eta / 2$ for each $t \in\left[t_{j}, t_{j+1}\right]$, 
(21) $H\left(\left(G(1), t_{1}\right)\right) \in W$.

Then $H\left(\left(G(l), t_{l}\right)\right) \in W$.

Proof. The proof is by induction over $j(j=1,2, \ldots, l)$. For $j=1$ the lemma follows from (21). Hence it is sufficient to show that if $H\left(G(j-1), t_{j-1}\right) \in W$, then $H\left(G(j), t_{j}\right) \in W$. We will consider the following two cases corresponding to (20)(ii) and (20)(iii) respectively.

Case 1. $F(j-1)=F(j)$ and $\left|t_{j}-t_{j-1}\right|<\eta / 2$. By (16), (18) and (20)(i), we have $H\left(G(j), t_{j}\right) \in B\left(z_{1}, \varepsilon\right)$. By (19) and (20)(ii) there exists a continuum $P_{j} \subset X \times I$ such that $\left(G(j-1), t_{j-1}\right),\left(G(j), t_{j}\right) \in P_{j}$ and $\operatorname{diam}\left(P_{j}\right)<\eta$. Hence it follows from the definition of $W$ and (16) that $H\left(G(j), t_{j}\right) \in W$.

Case 2. We will first show that $H\left(G(j-1), t_{j}\right) \in W$. Let $p_{1}, p_{2}, \ldots, p_{a}$ be a partition of $\left[t_{j-1}, t_{j}\right]$ such that $p_{1}=t_{j-1}, p_{a}=t_{j}$ and $\left|p_{i-1}-p_{i}\right|<\eta$ for each $i \in[2, a]$. Since $H\left(G(j-1), p_{1}\right) \in W$, it is sufficient to show that if $H\left(G(j-1), p_{i-1}\right) \in W$, then $H\left(G(j-1), p_{i}\right) \in W(i=2, \ldots, a)$. By (16), (18) and (20)(iii), $H\left(G(j-1), p_{i}\right) \in B\left(z_{1}, \varepsilon\right)$. Since $\left|p_{i}-p_{i-1}\right|<\eta$, there exists a continuum $Q_{i} \subset X \times I$ such that $\left(G(j-1), p_{i-1}\right),\left(G(j-1), p_{i}\right) \in Q_{i}$ and $\operatorname{diam}\left(Q_{i}\right)$ $<\eta$. Hence $H\left(G(j-1), p_{i}\right) \in W(i=1,2, \ldots, a)$, in particular, $H\left(G(j-1), p_{a}\right)$ $=H\left(G(j-1), t_{j}\right) \in W$.

By (18) and (20)(i), $H\left(G(j), t_{j}\right) \in B\left(z_{1}, \varepsilon\right)$, and by (19) and the above, $H\left(G(j), t_{j}\right)$ $\in W$.

Hence $H\left(G(j), t_{j}\right) \in W$ for each $j=1,2, \ldots, l$. In particular, $H\left(G(l), t_{l}\right) \in W$ and the proof is complete.

6. Contractible fans. In this section we establish some of the main results of this paper. Theorem 6.1 will be used in the proof of Theorem 6.2 which gives a solution to a problem raised in [3].

6.1. ThEOREM. Let $X$ be a contractible fan, and let $v$ be the vertex of $X$; then $X$ is locally connected at $v$.

Proof. Suppose, on the contrary, that $H: X \times I \rightarrow X$ is a contraction of $X$, and $X$ is not locally connected at $v$. By Theorem $2.2, X$ does not have property $P$. Hence there exists a sequence $\left\{v_{i}\right\}(i=1,2, \ldots)$ in $X$ converging to $v$ such that $\operatorname{Ls}\left[v, v_{i}\right]=K \neq\{v\}$. We may assume that

(1) $\operatorname{Lim}\left[v, v_{i}\right]=K \neq\{v\}$.

Let $s>0$ and let $V$ be a neighborhood of $v$ such that $\overline{B(v, s)} \subset V$.

Claim 1. There exists a number $\nu>0$ such that for each $i=1,2, \ldots$, at most $\nu$-many components of $\left[v, v_{i}\right] \backslash B(v, s)$ intersect $X \backslash V$.

Proof of Claim 1. Let $r>0$ such that $\overline{B(v, s)} \subset B(v, r) \subset \overline{B(v, r)} \subset V$, and let $\rho$ be the uniform continuity measure of $H$ for $r-s$, such that $\rho<s$. Suppose Claim 1 is false. Then there exists an index $i_{0}$ such that $d\left(v, v_{i_{0}}\right)<\rho$, and if $2 b$ is the number of components of $\left[v, v_{i_{0}}\right] B(v, s)$ intersecting $X \backslash V$, then $b \geqslant 1 / \rho$. Moreover we may assume that $H\left(v_{i_{0}}, t\right)=v$ for some $t \in I$. There exists a sequence $v_{i_{0}}=x_{1}>x_{2}>\cdots>x_{2 b}$ of points of $\left[v, v_{i_{0}}\right]$ such that

(2) $x_{2 j-1} \in B(v, s)$ and $x_{2 j} \in X \backslash V(j=1, \ldots, b)$. 
Let $t_{2 b}=\inf \left\{t \in I \mid H\left(v_{i_{0}}, t\right)=v\right\}$ and $t_{j}=\sup \left\{t \in\left[0, t_{2 b}\right] \mid H\left(v_{i_{0}}, t\right)=x_{j}\right\} \quad(j=$ $1,2, \ldots, 2 b-1)$.

Then $t_{1}<t_{2}<\cdots<t_{2 b}$ and since $b \geqslant 1 / \rho$, there exists an index $j$ such that $\left|t_{j+1}-t_{j}\right|<\rho$. Hence

(3) $d\left(H\left(v_{i_{0}}, t_{j}\right), H\left(v_{i_{0}}, t_{j+1}\right)\right)<r-s$.

Since $H\left(v_{i_{0}}, t_{j}\right)=x_{j}$ and $H\left(v_{i_{0}}, t_{j+1}\right)=x_{j+1}$, we have by (2), $d\left(H\left(v_{i_{0}}, t_{j}\right), H\left(v_{i_{0}}, t_{j+1}\right)\right)$ $>r-s$, contrary to (3) and Claim 1 is proved.

A point $a \in K$ is called an endpoint (of $K$ ) provided $a$ is an endpoint of every arc contained in $K$. If $K=[v, a]$ for some $a \in X$, then it follows easily that $X$ is of type $N$ between the points $a$ and $v$, and hence $X$ is not contractible (cf. Theorem 2.1), contrary to the assumptions. Hence we may assume that $K$ contains at least two endpoints $a$ and $b$ different from $v$. Let $t_{a}=\inf \{t \in I \mid H(v, t)=a\}$ and $t_{b}=\inf \{t \in I \mid H(v, t)=b\}$ and assume that $t_{a}<t_{b}$ (notice that $t_{a}$ and $t_{b}$ exist by Lemma 5.3). By Corollary 5.4, there exists a neighborhood $U$ of $v$ such that $U \cap K$ is connected and $a$ and $b$ belong to the interior of $X \backslash U$. Let $t^{*}=\sup \{t \in$ $\left.\left[0, t_{a}\right] \mid H(v, t) \in U\right\}$. Choose a point $z \in[v, a]$ and a number $\varepsilon_{1}>0$ such that

(4) $B\left(z, 5 \varepsilon_{1}\right) \cap\left[U \cup\{a\} \cup H\left(\{v\} \times\left[0, t^{*}\right]\right)\right]=\varnothing$,

(5) $B\left(z, 3 \varepsilon_{1}\right) \cap K \subset[v, a]$.

Let $\eta_{1}$ be the uniform continuity measure of $H$ for $\varepsilon_{1}$ and let $V$ be a neighborhood of $v$ such that

(6) $\bar{V} \subset U \cap B\left(v, \eta_{1} / 9\right)$ and $V \cap K$ is connected.

Since $K$ is locally connected and $V \cap K$ is connected, it follows that $K \backslash V$ has finitely many components $K_{1}, K_{2}, \ldots, K_{\gamma}$. Let

(7) $y_{j}=\inf \left\{x \in K_{j}\right\}$ and $e_{j}=\sup \left\{x \in K_{j}\right\}(j=1, \ldots, \gamma)$.

Choose a finite sequence of distinct points $\left\{x_{i}\right\}(i=1,2, \ldots, m)$ in $(K \backslash V) \cup$ $\{v\}$ such that

(8) $x_{0}=v, x_{c}=z, x_{n}=a$ and $x_{s}=b$ for some $c, n, s \in[1, m]$, and for each $j \in[1, \gamma]$, there exist $p, q \in[1, m]$ such that $x_{p}=y_{j}$ and $x_{q}=e_{j}$.

(9) If $\left[x_{p}, x_{q}\right] \cap\left[\cup_{i=1}^{m}\left\{x_{i}\right\} \backslash\left\{x_{p}, x_{q}\right\}\right]=\varnothing$ for some $p, q \in[1, m]$, then $\operatorname{diam}\left(\left[x_{p}, x_{q}\right]\right)<\eta_{1} / 4$.

(10) Let $t_{j}=\inf \left\{t \in I \mid H(v, t)=x_{j}\right\} \quad(j=1,2, \ldots, m)$; then $0=t_{0}<t_{1}$ $<\cdots<t_{m}$.

Choose a number $\alpha>0$ such that

(11) $\alpha<\frac{1}{3} \min \left\{\varepsilon_{1}, \eta_{1} / 5, \min _{i \neq j}\left\{d\left(x_{i}, x_{j}\right)\right\}\right\}$,

(12) $H\left(\{v\} \times\left[0, t_{j-1}\right]\right) \cap B\left(x_{j}, 2 \alpha\right)=\varnothing(j=1, \ldots, m)$.

Let $U_{j}$ be a neighborhood of $x_{j}(j=1, \ldots, m)$ such that $\bar{U}_{j} \subset B\left(x_{j}, \alpha / 2\right)$ and $U_{j} \cap K$ is connected. Let

(13) $\varepsilon_{2}=\frac{1}{2} \min \left\{\alpha, \min _{j=1, \ldots, m}\left\{d\left(x_{j}, X \backslash U_{j}\right)\right\}\right\}$ and let $\eta_{2}$ be the uniform continuity measure of $H$ for $\varepsilon_{2}$ and $U_{0}$ a neighborhood of $v$ such that

(14) $U_{0} \subset B\left(v, \eta_{2} / 2\right) \subset \overline{B\left(v, \eta_{2}\right)} \subset V$ and $U_{0} \cap K$ is connected.

Since $K$ is locally connected and $U_{0} \cap K$ is connected, we have that $K \backslash U_{0}$ has at most finitely many components $D_{1}, D_{2}, \ldots, D_{k}$. Let

$$
\beta=\frac{1}{2} \min \left\{\eta_{2}, \frac{1}{3} \min _{i \neq j}\left\{d\left(D_{i}, D_{j}\right)\right\}\right\}
$$


and $n_{0}>0$ such that

(15) $v_{i} \in B(v, \beta),\left[v, v_{i}\right] \subset B(K, \beta)$ and $K \subset B\left(\left[v, v_{i}\right], \beta\right)$ for all $i>n_{0}$. Hence in particular, $\left[v, v_{i}\right] \cap U_{n} \neq \varnothing \neq\left[v, v_{i}\right] \cap U_{s}$ for $i>n_{0}$.

Let $A$ and $B$ be subsets of $X$, then we say that $A<B$ provided $x<y$ for each $x \in A$ and each $y \in B$. By Lemma 5.5, there exists an index $n_{1}>n_{0}$ such that for each component $C$ of $\left[v, v_{i}\right] \backslash U_{0}$, at most two components of $C \cap B\left(x_{j}, \alpha\right)$ intersect $\bar{U}_{j}(j=1,2, \ldots, m)$, and by Claim 1 , there are at most finitely many components of $\left[v, v_{i}\right] \backslash U_{0}$ that intersect $X \backslash V$. We will for convenience assume that $n_{1}=1$, where we relabel if necessary.

Let $K(i, 0)$ be the first component of $\left[v, v_{i}\right] \backslash U_{0}$ that intersects $\bar{U}_{n}$, i.e. if $C$ is any component of $\left[v, v_{i}\right] \backslash U_{0}$ such that $C<K(i, 0)$, then $C \cap \bar{U}_{n}=\varnothing(i=1,2, \ldots)$. Label all other components of $\left[v, v_{i}\right] \backslash U_{0}, K(i, h)(h= \pm 1, \pm 2, \ldots)$ such that .. $<K(i,-2)<K(i,-1)<K(i, 0)<K(i, 1)<K(i, 2)<\cdots(i=$ $1,2, \ldots)$. Let $h_{i}$ and $h_{i}^{\prime}$ be integers such that $h_{i}<h<h_{i}^{\prime}$ for each component $K(i, h)$, where $h_{i}$ is maximal and $h_{i}^{\prime}$ is minimal with respect to this property $(i=1,2, \ldots)$.

In each component $C$ of $K(i, h) \cap B\left(x_{j}, \alpha\right)$ that intersects $\bar{U}_{j}$, choose one point $x(i, h, j, w, e) \in \bar{U}_{j}(j=1, \ldots, m)$ and choose one point $x(i, h, 0,0, e) \in \bar{U}_{0}$ between $K(i, h)$ and $K(i, h+1)\left(h=h_{i}, \ldots, h_{i}^{\prime}-1\right)$, where $w=0$ if only one component of $K(i, h) \cap B\left(x_{j}, \alpha\right)$ intersects $\bar{U}_{j}$, and $x(i, h, j, 1, e)>x\left(i, h, j, 2, e^{\prime}\right)$ otherwise $(j=1, \ldots, m) ; e=1$ for the point(s) chosen in $K(i, 0) \cap \bar{U}_{n}$ and $e$ represents the number of labeled points on $\left[x(i, h, j, w, e), x\left(i, 0, n, w^{\prime}, 1\right)\right]$ otherwise, where either $w^{\prime} \in\{0,1\}$ and $h>0$, or $h=0$ and $w=1$; or $w^{\prime} \in\{0,2\}$ and $h<0$, or $h=0$ and $w=2$.

Let $p_{i}-1\left(q_{i}-1\right)$ be the maximum of the numbers $e$ such that there exists a point $x(i, h, j, w, e)$ with $h<0\left(h>0\right.$, respectively). Put $x\left(i, h_{i}-1,0,0, p_{i}\right)=v$ and $x\left(i, h_{i}^{\prime}, 0,0, q_{i}\right)=v_{i}$. Notice that the quartets of numbers $i, h, w, e$ and $i, h, j, w$ each uniquely determine a point $x(i, h, j, w, e)$ in $X$.

Claim 2. Let $a_{i}=x(i, h, j, w, e)$ and $b_{i}=x\left(i, h^{\prime}, j^{\prime}, w^{\prime}, e^{\prime}\right)$. Suppose $j^{*}$ is a natural number such that $j^{*}<\min \left\{j, j^{\prime}\right\}$, and $h<h^{\prime}$. Then there exists a point $c_{i}=x\left(i, h^{*}, j^{*}, w^{*}, e^{*}\right)$ such that $a_{i}<c_{i}<b_{i}$.

Proof of Claim 2. Suppose Claim 2 is false; then

(16) $\left[a_{i}, b_{i}\right] \cap \bar{U}_{j^{*}}=\varnothing$.

Since $h<h^{\prime}$, there exists a point $v_{i}^{\prime} \in \bar{U}_{0}$ such that $a_{i}<v_{i}^{\prime}<b_{i}$. Recall that $H\left(v, t_{j^{*}}\right)=x_{j^{*}}$ and hence by (13) and (14), $H\left(v_{i}^{\prime}, t_{j^{*}}\right) \in B\left(x_{j^{*}}, \varepsilon_{2}\right) \subset U_{j^{*}}$. Yence by (16), there exists $t^{\prime}<t_{j^{*}}$ such that either $H\left(v_{i}^{\prime}, t^{\prime}\right)=a_{i}$ or $H\left(v_{i}^{\prime}, t^{\prime}\right)=b_{i}$. We may assume that $H\left(v_{i}^{\prime}, t^{\prime}\right)=a_{i} \in \bar{U}_{j} \subset B\left(x_{j}, \alpha / 2\right)$, then

$$
\begin{aligned}
d\left(H\left(v, t^{\prime}\right), x_{j}\right) & <d\left(H\left(v, t^{\prime}\right), H\left(v_{i}^{\prime}, t^{\prime}\right)\right)+d\left(H\left(v_{i}^{\prime}, t^{\prime}\right), x_{j}\right) \\
& <\varepsilon_{2}+\alpha / 2<3 \alpha / 2,
\end{aligned}
$$

contrary to (12) since $t^{\prime}<t_{j^{*}}<t_{j-1}$, and the proof of Claim 2 is complete.

Claim 3. We may assume that for each point $x(i, h, j, w, e)$ with $h<0$ and $e>1$, the following two conditions are satisfied.

(i) $j<n$. 
(ii) There exists a point $x\left(i, h^{\prime}, j, w^{\prime}, e^{\prime}\right)$ with $h^{\prime}>0$ or $h^{\prime}=0$ and $w^{\prime} \in\{0,1\}$ and such that for each point $x\left(i, h^{\prime \prime}, j^{\prime \prime}, w^{\prime \prime}, e^{\prime \prime}\right)$, where $h^{\prime \prime}>0$ or $h^{\prime \prime}=0$ and $w^{\prime \prime} \in\{0,1\}$, and $1<e^{\prime \prime} \leqslant e^{\prime}$, we have $j^{\prime \prime}<n$.

Proof of Claim 3. We will first show that (i) is true. Let

$$
j_{0}=\max \{j \in[1, m] \mid \text { there exists a point } x(i, h, j, w, e) \text { with } h<0\},
$$

and suppose $j_{0} \geqslant n$. Since $K(i, h) \cap \bar{U}_{n}=\varnothing$ if $h<0$, we have $j_{0}>n$.

Define $U^{*}, z^{*}, \varepsilon_{1}^{*}, \eta_{1}^{*}, V^{*}, m^{*},\left\{x_{i}^{*}\right\}\left(i=1, \ldots, m^{*}\right), U_{j}^{*}, \alpha^{*}, \varepsilon_{2}^{*}, \eta_{2}^{*}, \beta^{*}, n_{0}^{*}, n_{1}^{*}$, and the labelings $K^{*}(i, h), x^{*}(i, h, j, w, e)$ as $U, z, \varepsilon_{1}, \eta_{1}, V, m,\left\{x_{i}\right\}(i=$ $1,2, \ldots, m), U_{j}, \alpha, \varepsilon_{2}, \eta_{2}, \beta, n_{0}, n_{1}$, and the labelings $K(i, h)$ and $x(i, h, j, w, e)$, the only difference being that the points $a=x_{n}$ and $b=x_{s}$ are replaced by the points $x_{j_{0}}$ and $a$ respectively and we require moreover that $\varepsilon_{1}^{*}<\varepsilon_{1}, \eta_{1}^{*}<\eta_{1}, \varepsilon_{2}^{*}<\varepsilon_{2}$, $\eta_{2}^{*}<\eta_{2}$ and $U_{n^{*}}^{*} \subset U_{n}$. Suppose $x_{j_{0}^{*}}^{*}=x_{j_{0}}$ and $x_{n^{*}}^{*}=x_{n}=a$, then $t_{n^{*}}=t_{n}, t_{j_{0}}=t_{j_{0}^{*}}$. We claim that (i) is true for the new labeling $x^{*}(i, h, j, w, e)$. We will prove an even stronger statement by showing that if $x^{*}(i, h, j, w, e)$ is a point of the new labeling with $h<0$, then $j<n^{*}<j_{0}^{*}$.

Suppose this is not the case. Then, as above, there exists a point $a_{i}=$ $x^{*}(i, h, j, w, e)$ with $h<0$ and $j>n^{*}$. Let $b_{i}=x^{*}\left(i, 0, j_{0}^{*}, w, e\right)$, where $w \in\{0,2\}$, i.e. $b_{i}$ is a point in the first component of $\left[v, v_{i}\right] \backslash U_{0}^{*}$ that intersects $U_{j \neq}^{*}$. Since $j_{0}^{*}>n^{*}$ and $a=x_{n}=x_{n^{*}}^{*}$ is an endpoint of $K$, we have $b_{i}<K(i, 0)$. Also, since $a_{i} \in K^{*}(i, h)$ with $h<0$, we have $a_{i} \in K^{*}(i, h)<K^{*}(i, 0)<K(i, 0)$. Hence by the definition of $K(i, 0)$, we have $\left[a_{i}, b_{i}\right] \cap \bar{U}_{n}=\varnothing$. It follows that $\left[a_{i}, b_{i}\right] \cap \bar{U}_{n^{*}}^{*}=\varnothing$, contrary to Claim 2 for the new labeling, since $n^{*}<\min \left\{j, j_{0}^{*}\right\}$.

We will show next that if (i) holds for the original labeling, then (ii) holds for this labeling, and if (i) does not hold for the original labeling, then (ii) holds for the new labeling as defined above.

Suppose first that (i) holds for the original labeling. Let $x(i, h, j, w, e)$ be a point with $h<0$; then $j<n$. Recall that $b=x_{s}$ and $s>n$. By $(15),\left[v, v_{i}\right] \cap \bar{U}_{s} \neq \varnothing$. Hence there exists a point $\hat{b}=x(i, \hat{h}, s, \hat{w}, \hat{e})$, with $h>0$. By Claim 2, there exists a point $x\left(i, h^{\prime}, j, w^{\prime}, e^{\prime}\right)$ between the points $\hat{b}$ and $q=x\left(i, 0, n, w_{1}, e_{1}\right)$, where $w_{1} \in\{0,1\}$, and we may assume that $e^{\prime}$ is minimal with respect to these properties. Suppose there exists a point $p=x\left(i, h^{\prime \prime}, j^{\prime \prime}, w^{\prime \prime}, e^{\prime \prime}\right)$ with $h^{\prime \prime}>0, w^{\prime \prime} \in\{0,1\}$, $j^{\prime \prime}>n$ and $1<e^{\prime \prime}<e^{\prime}$. Since $a=x_{n}$ is an endpoint of $K$, we have $h^{\prime \prime}>0$. Hence by Claim 2, there exists a point $x\left(i, h^{*}, j, w^{*}, e^{*}\right)$ between the points $p$ and $q$, contradicting the fact that $e^{\prime}$ is minimal. This contradiction shows that (ii) holds for the original labeling.

Suppose next that (i) does not hold for the original labeling. Define a new labeling as above. Let $x^{*}(i, h, j, w, e)$ be a point with $h<0$. It follows from the above that $j<n^{*}$. Let $p=x^{*}\left(i, 0, j_{0}^{*}, w_{1}, e_{1}\right)$ and $q=x^{*}\left(i, h^{*}, n^{*}, w^{*}, e^{*}\right)$. Since $j_{0}^{*}>n^{*}, p<q$ and $a$ is an endpoint of $K$, we have $h^{*}>0$. Hence by Claim 2, there exists a point $x^{*}\left(i, h^{\prime}, j, w^{\prime}, e^{\prime}\right)$, between the points $p$ and $q$, and we may assume that $e^{\prime}$ is minimal with respect to this property. Suppose there exists a point $y=x^{*}\left(i, h^{\prime \prime}, j^{\prime \prime}, w^{\prime \prime}, e^{\prime \prime}\right)$ with $h^{\prime \prime}>0, j^{\prime \prime}>j_{0}^{*}$ and $1<e^{\prime \prime}<e^{\prime}$. Then by Claim 2, there exists a point $x^{*}(i, h, j, w, e)$, contrary to the fact that $e^{\prime}$ is minimal. This 
contradiction completes the proof of Claim 3. For convenience we will assume that Claim 3 is true for the original labeling.

Let $A_{i}$ be the set of all points $x(i, h, j, w, e)$ such that either $h<0$, or $h=0$ and $w \in\{0,2\}$, and let $B_{i}$ be the set of all points $x(i, h, j, w, e)$ with either $h>0$, or $h=0$ and $w \in\{0,1\}$. Recall that $e \in\left[1, p_{i}\right]$ for all points belonging to the set $A_{i}$ and $e \in\left[1, q_{i}\right]$ for all points belonging to $B_{i}$, where $p_{i}$ and $q_{i}$ are minimal with respect to these properties. Let $N$ denote the set of natural numbers. Define functions $f_{i}:\left[1, p_{i}\right] \rightarrow N$ and $g_{i}:\left[1, q_{i}\right] \rightarrow N$ by

$$
\begin{array}{ll}
f_{i}(e)=j, & \text { if } x(i, h, j, w, e) \in A_{i}, \\
g_{i}(e)=j, & \text { if } x(i, h, j, w, e) \in B_{i} .
\end{array}
$$

It follows from the construction of the labeling that $f_{i}$ and $g_{i}$ are well defined.

ClaIm 4. $f_{i}$ and $g_{i}$ are uniform of order $n$.

Proof of Claim 4. We will verify conditions (1) and (2) of §3. Suppose first that condition (2) does not hold for some $f_{i}$; i.e. there exists a number $t>0$ and $e \in\left[1, p_{i}\right]$ such that

$$
f_{i}\left(e^{\prime}\right) \geqslant f_{i}(e)=t \quad \text { if }\left|e-e^{\prime}\right|<1 .
$$

It follows from the definition of the labeling $x(i, h, j, w, e)$, that $f_{i}(2)<f_{i}(1)$. By definition of $p_{i}$, we have $f_{i}\left(p_{i}\right)=0$. Hence we may assume $e \in\left[2, p_{i}\right)$, and therefore that $e-1, e+1 \in\left[1, p_{i}\right]$. Let $f_{i}(e-1)=t^{\prime}, f_{i}(e+1)=t^{\prime \prime}, a=x\left(i, h^{\prime}, t^{\prime}, w^{\prime}, e-\right.$ $1), b=x(i, h, j, w, e)$ and $c=x\left(i, h^{\prime \prime}, t^{\prime \prime}, w^{\prime \prime}, e+1\right)$. Then, since $t^{\prime}, t^{\prime \prime}>t>0$, all three points $a, b$ and $c$ belong to the same component $K(i, h)$ of $\left[v, v_{i}\right] \backslash U_{0}$, i.e. $h=h^{\prime}=h^{\prime \prime}$. It follows from the definition of $e$ that $a>b>c$. Let $p=$ $x\left(i, h-1,0,0, e_{1}\right)$ and $q=x\left(i, h, 0,0, e_{2}\right)$, i.e. $p, q \in U_{0}$ and $p<c, q>a$. Let $C(i, 2)$ be the component of $K(i, h) \cap B\left(x_{t}, \alpha\right)$ that contains the point $b$. Since $x_{t^{\prime}}>x_{t}$, we have by (15) that there exists a component $C(i, 1)$ of $K(i, h) \cap B\left(x_{t}, \alpha\right)$ such that $C(i, 1) \cap \bar{U}_{t} \neq \varnothing$ and $p<C(i, 1)<c<C(i, 2)$. Similarly, there exists a component $C(i, 3)$ of $K(i, h) \cap B\left(x_{t}, \alpha\right)$ such that $C(i, 3) \cap \bar{U}_{t} \neq \varnothing$, and $C(i, 2)<$ $a<C(i, 3)<q$. We conclude that at least three components of $K(i, h) \cap B\left(x_{t}, \alpha\right)$ intersect $\bar{U}_{t}$, contrary to Lemma 5.5 .

We will show next that (1) holds for the functions $f_{i}$. Let $t>0, e, e-1 \in\left[1, p_{i}\right]$ and suppose $f_{i}(e-1)<f_{i}(e)=t$. Let $v=x_{h(u)}<\cdots<x_{h(2)}<x_{h(1)}<x_{h(0)}=x_{t}$ be all members of the partition $\left\{x_{i}\right\}(i=1,2, \ldots, m)$ that belong to the arc $\left[v, x_{t}\right]$. By using (2) of $\$ 3$ repeatedly, we have $0=f_{i}(e-v)<\cdots<f_{i}(e-1)<f_{i}(e)$ for some positive integer $v$. It follows from (15) and the definition of the function $f_{i}$ that $f_{i}(e-s)=h(s)(s=0,1, \ldots, u)$ and $u=v$. In case $e, e+1 \in\left[1, p_{i}\right]$ and $f_{i}(e+1)<f_{i}(e)$, then it follows as above that $f_{i}(e+s)=h(s)(s=0,1, \ldots, u)$. Similarly, it follows that the function $g_{i}$ satisfies (1) and (2), and the proof of Claim 4 is complete.

Clam 5. Let $a_{i}=x(i, h, p, w, e)$ and $b_{i}=x\left(i, h^{\prime}, q, w^{\prime}, e+1\right)$ be two points of $\left[v, v_{i}\right]$ such that one of the following two conditions is satisfied.

(i) $h>0$ or $h=0$ and $w \in\{0,1\}$, and $h^{\prime}>0$ or $h^{\prime}=0$ and $w^{\prime} \in\{0,1\}$,

(ii) $h<0$ or $h=0$ and $w \in\{0,2\}$, and $h^{\prime}<0$ or $h^{\prime}=0$ and $w^{\prime} \in\{0,2\}$.

Then $\operatorname{diam}\left(\left[a_{i}, b_{i}\right]\right)<\eta_{1} / 2$. Moreover if two components of $K(i, 0) \cap B\left(x_{n}, \alpha\right)$ 
intersect $\bar{U}_{n}$, then

$$
\operatorname{diam}([x(i, 0, n, 1,1), x(i, 0, n, 2,1)])<\eta_{1} / 2 .
$$

Proof of Claim 5. By definition of $e$ and (15), we have

$$
\left[x_{p}, x_{q}\right] \cap\left[\bigcup_{i=1}^{m}\left\{x_{i}\right\} \backslash\left\{x_{p}, x_{q}\right\}\right]=\varnothing .
$$

Hence by (9), diam $\left(\left[x_{p}, x_{q}\right]\right)<\eta_{1} / 4$. We consider three cases as follows.

Case 1. $p=q=0$. By (7), (8), (15) and the above we have

$$
\left[a_{i}, b_{i}\right] \subset B(V, \beta) \subset B\left(v, \beta+\eta_{1} / 5\right) \subset B\left(v, \eta_{1} / 4\right) .
$$

Hence $\operatorname{diam}\left(\left[a_{i}, b_{i}\right]\right)<\eta_{1} / 2$.

Case 2. Either $p=0$ or $q=0$. We may assume $p=0$ and $q \neq 0$. Then by (7), (8), (15) and the above,

$$
\left[a_{i}, b_{i}\right] \subset V \cup B\left(x_{q}, \alpha\right) \subset B\left(v, \eta_{1} / 5\right) \cup B\left(x_{q}, \alpha\right)
$$

and hence by (11),

$$
\operatorname{diam}\left(\left[a_{i}, b_{i}\right]\right)<2 \eta_{1} / 5+\alpha<\eta_{1} / 2 .
$$

Case 3. $p, q>0$. Then by (7), (8), (15) and the above,

$$
\left[a_{i}, b_{i}\right] \subset B\left(\left[x_{p}, x_{q}\right], \beta\right) \cup B\left(x_{p}, \alpha\right) \cup B\left(x_{q}, \alpha\right)
$$

and hence by (11), (13) and the definition of $\beta$,

$$
\operatorname{diam}\left(\left[x_{p}, x_{q}\right]\right) \leqslant \eta_{1} / 4+2 \beta+\alpha+\alpha<3 \alpha+\eta_{1} / 4<\eta_{1} / 2
$$

The proof that $\operatorname{diam}([x(i, 0, n, 1,1), x(i, 0, n, 2,1)])<\eta_{1} / 3$, being a duplicate of Case 3 , is omitted, and the proof of Claim 5 is complete.

Since $\{v\} \times I$ is a locally connected continuum and the mapping $H \mid\{v\} \times I$ is continuous, we have that $H(\{v\} \times I)$ is locally connected. Hence there exists an index $n_{2}$ such that

(17) $x(i, 0, c, 2, e) \notin H(\{v\} \times I)$ for $i \geqslant n_{2}$.

Recall that $z=x_{c}$ and $a=x_{n}$. As $H(X \times\{1\})$ is one point, there exists an index $n_{3}>n_{2}$ such that

(18) $x(i, 0, c, 2, e) \notin H(X \times\{1\})$ for $i \geqslant n_{3}$.

Let $z_{1}=x\left(n_{3}, 0, c, 2, s\right), z_{2}=x\left(n_{3}, 0, c, 1, s\right), a_{1}=x\left(n_{3}, 0, n, w, 1\right)$ and $a_{2}=$ $x\left(n_{3}, 0, n, w^{\prime}, 1\right)$, where $w \in\{0,2\}$ and $w^{\prime} \in\{0,1\}$. From here on we will take $i=n_{3}$, and we omit the subscript $i$ if no confusion is possible. In particular, $f_{n_{3}}=f$, $g_{n_{3}}=g, A_{n_{3}}=A, B_{n_{3}}=B$ and $x\left(n_{3}, h, j, w, e\right)=x(h, j, w, e)$.

Let $C$ be the component of $H^{-1}\left(z_{1}\right) \cap\left[v, a_{1}\right] \times I$ that contains the point $\left(z_{1}, 0\right)$. By (17) and (18),

(19) $C \cap[(\{v\} \times I) \cup(X \times\{1\})]=\varnothing$.

ClaIm 6. $C \cap\left(\left\{a_{1}\right\} \times I\right) \neq \varnothing$.

Proof of Claim 6. Suppose Claim 6 is false; then by (19),

(20) $C \cap\left[\{v\} \times I \cup\left[v, a_{1}\right] \times\{1\} \cup\left\{a_{1}\right\} \times I\right]=\varnothing$.

Since $X$ is hereditarily unicoherent, we have

(21) each arc joining the points $v$ and $a_{1}$ contains the point $z_{1}$. 
Condition (20) is a replica of condition (8) in [9], where $q_{k}, B_{k}$ and $q_{k}^{\prime}$ are replaced by $v,\left[v, a_{1}\right]$ and $a_{1}$, respectively. In exactly the same way as (8) led to a contradiction with (5), now (20) leads to a contradiction with (21). This contradiction completes the proof of Claim 6.

Hence $C \cap\left(\left\{a_{1}\right\} \times I\right) \neq \varnothing$. Let $\left(a_{1}, t^{*}\right)$ be a point of $C \cap\left(\left\{a_{1}\right\} \times I\right)$. Put $y_{e}=x(h, j, w, e)$ if $x(h, j, w, e) \in A(e=1,2, \ldots, p)$. Then by Claim $5, \mid y_{e}-$ $y_{e+1} \mid<\eta_{1} / 2$, and by definition, $y_{1}=a_{1}, y_{p}=v$ and $z_{1}=y_{u}$ for some $u \in(1, p)$. Hence $\left(y_{u}, 0\right)$ and $\left(y_{1}, t^{*}\right)$ are two points of $C \cap\left[y_{1}, y_{p}\right] \times I$.

Hence by Corollary 4.2 , there exists a finite set $R=[1, r]$, a function $F$ : $R \rightarrow[1, p]$ and a sequence $\left\{t_{k}\right\}(k=1,2, \ldots, r)$ of points in $I$ such that

(22) $F(1)=1, t_{1}=t^{*}, F(r)=u$ and $t_{r}=0$, and $\left(y_{F(k)}, t_{k}\right) \in C$ for each $k \in R$,

(23) $|F(k+1)-F(k)| \leqslant 1$ for each $k \in[1, r-1]$,

(24) for each $k, 1<k \leqslant r-1$, at least one of the following two is satisfied.

(i) $F(k)=F(k+1)$ and $\left|t_{k}-t_{k+1}\right|<\eta_{1} / 2$,

(ii) there exists a subcontinuum $C_{k}$ of $C$ such that $d\left(C_{k},\left(y_{F(k)}, t\right)\right)<\eta_{1} / 2$ for each $t \in\left[t_{k}, t_{k+1}\right]$.

Moreover we may assume

(25) $F(k) \geqslant 2$ for each $k \in[2, r]$.

Let

$$
q^{*}=\max \left\{e \in[1, q] \mid \text { if } e^{\prime} \in[2, e] \text {, then } g\left(e^{\prime}\right)<n\right\},
$$

$Q^{*}=\left[1, q^{*}\right]$ and $g^{*}=g \mid Q^{*}$. Recall that $z_{2}=x(0, c, 1, s)$ and hence $g(s)=c$. By Claim 3, $s \in Q^{*}$.

Claim 7. The functions $f: P \rightarrow N, g^{*}: Q^{*} \rightarrow N$ and $F: R \rightarrow P$, and the point $s \in Q^{*}$ satisfy all conditions of Lemma 3.1.

Proof of Claim 7. We will verify conditions (4)-(9) of Lemma 3.1.

(4) $f(1)=g^{*}(1)=n$ by the definition of $f$ and $g^{*}$, respectively. By Claim 3, $f(e)<n-1$ if $e \in P \backslash\{1\}$ and by the definition of $q^{*}, g^{*}(e)<n-1$ if $e \in$ $Q^{*} \backslash\{1\}$.

(5)' By (22), $F(1)=1$, and by (25) and Claim $3, f \circ F(k)<n-1$ for each $k \in[2, r]$. Since $z_{1}=x(0, c, 2, u)=y_{u}$, we have $f \circ F(r)=c=g^{*}(s)$.

(6)' follows from (23).

(7) By Claim 4, $f$ and $g$ are uniform of order $n$. By the definition of $q^{*}$, we have if $q^{*}+1 \in Q$, then $g\left(q^{*}+1\right)>g\left(q^{*}\right)$. Hence $f$ and $g^{*}$ are uniform of order $n$.

(8)' follows from Claim 2 and the definitions of $f$ and $g^{*}$.

(9)' We will verify (3)(i)-(3)(iii) of the definition of symmetry in $\$ 3$. Let $\left\{\left(t_{v}, k_{v}\right)\right\}$ $(v=0, \ldots, \gamma)$ be the local maxima sequence of $f \circ F$ and $\left\{a_{w}\right\} \quad(w=$ $0,1, \ldots, k(n))$ the sequence associated with the number $n$. By $(7)^{\prime}$ we have,

(26) $f(1+w)=a_{w}=g^{*}(1+w)$ and $a_{0}>a_{1}>\cdots>a_{k(n)}=0$.

Choose $v \in[-1, \gamma]$. By (ii) of Claim 3, there exists $j \in Q^{*}$ such that $g^{*}(j)=t_{v}$. If $v=-1$, put $j_{v}=1$. If $v \neq-1$, let $j_{v}=\min \left\{j \in Q^{*} \mid g^{*}(j)=t_{v}\right\}$. Then,

(i) $g^{*}\left(j_{v}\right)=t_{v}$. Suppose $j \in\left(s, j_{v}\right)$ and $g^{*}(j)>t_{v}$. Then by (26) and the definition of $t_{v}$, there exists $j^{\prime} \in\left(s, j_{v}\right)$ such that $g^{*}\left(j^{\prime}\right)=0$. Hence by (8)' there exists $j^{\prime \prime} \in\left(s, j_{v}\right)$ such that $g^{*}\left(j^{\prime \prime}\right)=t_{v}$, contrary to the definition of $j_{v}$. 
(ii) Choose $k \in\left(k_{v}, r\right)$. It follows from the definition of $k_{v}$ that $f \circ F(k)<t_{v}$. By (ii) of Claim 3, there exists $j \in Q^{*}$ such that $g^{*}(j)=f \circ F(k)$. Let $j^{*}=\min \{j \in$ $\left.Q^{*} \mid g^{*}(j)=f \circ F(k)\right\}$. Suppose $j \in\left(j^{*}, s\right)$; then as above, or, by using $(26), g^{*}(j)$ $<t_{v}$.

(iii) Choose $j \in\left(j_{v}, s\right)$. If $g^{*}(j)=a_{w}$ for some $w \in[0, k(n)]$, then (iii) follows from (26). If $g^{*}(j) \neq a_{w}$ for each $w \in[0, k(n)]$, then (iii) follows from (8)' and the proof of Claim 7 is complete.

Hence, by Lemma 3.1 , there exists a finite set $L=[1, l]$ and functions $G_{1}$ : $L \rightarrow R$ and $G_{2}: L \rightarrow Q^{*}$ such that

(27) $G_{1}(1)=G_{2}(1)=1, G_{1}(l)=r, G_{2}(l)=s$ and $f \circ F \circ G_{1}(m)=g^{*} \circ G_{2}(m)$ for each $m \in L$,

(28) $\left|G_{1}(m+1)-G_{1}(m)\right|<1$ and $\left|G_{2}(m+1)-G_{2}(m)\right|<1$ for each $m \in[1, l$ $-1]$.

Let ๆ: $L \rightarrow L$ be given by $\uparrow(m)=l+1-m$, i.e. $\|$ is an order reversing function. Define functions $\Phi: L \rightarrow X$ and $\Psi: L \rightarrow X$ by $\Phi(m)=$ $x\left(h, j, w, F \circ G_{1} \circ \uparrow(m)\right)$, where $h<0$ or $h=0$ and $w \in\{0,2\}$ and $\Psi(m)=$ $x\left(h, j, w, G_{2} \circ \bigcap(m)\right)$, where $h>0$ or $h=0$ and $w \in\{0,1\}$, respectively $(m=$ $\left.1,2, \ldots, l ; j=g^{*} \circ G_{2} \circ \eta(m)\right)$.

Clanm 8. The numbers $\varepsilon_{1}, \eta_{1}$ together with the points $z_{1}, z_{2} \in X$ and the functions $\Phi$ and $\Psi$ of $L$ into $X$ satisfy all the conditions of Lemma 5.6, where $C$ is the component of $H^{-1}\left(z_{1}\right) \cap\left[v, a_{1}\right] \times I$ containing the point $\left(z_{1}, 0\right)$.

Proof of Claim 8. We will verify conditions (16)-(21) of Lemma 5.6.

(16)' follows from the definition of $\eta_{1}$.

(17)' follows from (4) and (15).

(18) Since $f \circ F \circ G_{1} \circ \eta(m)=g^{*} \circ G_{2} \circ \eta(m)$ for each $m \in L$, we have $\Phi(m)$, $\Psi(m) \in \bar{U}_{j} \subset \overline{B\left(x_{j}, \frac{1}{2} \alpha\right)}$ and (18)' follows from (11).

(19)' follows from (28) and Claim 5.

(20)' By (22), (23), (24) and (28), the sequence $\left\{t_{G_{1} \circ 9(m)}\right\}$ and the function $\Phi$ satisfy (i)-(iii).

(21) $H\left(\Psi(1), t_{G_{1} \circ \uparrow(1)}\right)=H\left(z_{2}, 0\right)=\left(z_{2}, 0\right) \in W$ by the definition of $\Psi,(22)$, (27) and the definition of $W$.

Hence, by Lemma 5.6, $H\left(\Psi(l), t_{G_{1} \circ 9(l)}\right)=H\left(a_{2}, t^{*}\right) \in W, H\left(a_{1}, t^{*}\right)=z_{1}$. Since $z_{1}$ and $z_{2}$ are $\varepsilon_{1}$-related (cf. (15), §4.1), we have $\operatorname{diam}\left(H\left(\left[a_{1}, a_{2}\right] \times\left\{t^{*}\right\}\right)\right)>\varepsilon_{1}$. By Claim 5, diam $\left(\left[a_{1}, a_{2}\right]\right)<\eta_{1} / 2$, and hence $\operatorname{diam}\left(H\left(\left[a_{1}, a_{2}\right] \times\left\{t^{*}\right\}\right)\right)<\varepsilon_{1}$. This contradiction completes the proof of Theorem 6.1.

The following theorem gives a solution to problem 786 raised in [3].

6.2. Theorem. Let $X$ be a contractible fan; then $X$ is embeddable in the plane.

Proof. By Theorem 6.1, $X$ is locally connected at the vertex $v$ of $X$. Hence by Theorem 5.2 of [10], $X$ is embeddable in the plane.

Acknowledgements. The author wishes to thank Professor A. Lelek for his encouragement and advice during the preparation of this paper. This research was supported by a Graduate Fellowship from Wayne State University, Detroit, Michigan. 


\section{REFERENCES}

1. David P. Bellamy and J. J. Charatonik, The set function $T$ and contractibility of continua, Bull. Acad. Polon. Sci. Sér. Sci. Math. Astronom. Phys. 25 (1977), 47-49.

2. K. Borsuk, A countable broom which can not be imbedded in the plane, Colloq. Math. 10 (1963), 233-236.

3. J. J. Charatonik and C. A. Eberhart, On contractible dendroids, Colloq. Math. 25 (1972), 89-98.

4. J. J. Charatonik, Problems and remarks on contractibility of curves, General Topology and Its Relations to Modern Analysis and Algebra. IV, Part B, Assoc. of Czechoslovak Math. and Phys. (Proc. Fourth Prague Topological Sympos., Prague, 1976), Prague, 1977.

5. J. J. Charatonik and Z. Grabowski, Homotopically fixed arcs and the contractibility of dendroids, Fund. Math. 100 (1978), 229-237.

6. G. B. Graham, On contractible fans, Doctoral Dissertation, Univ. of California, Riverside, Calif., 1977.

7. K. Kuratowski, Topology, Vol. II, Academic Press, New York, 1968.

8. J. Mioduszewski, Everywhere oscillating functions, extension of the uniformization and homogeneity of the pseudo-arc, Fund. Math. 56 (1964), 131-155.

9. Lex G. Oversteegen, Non-contractibility of contimua, Bull. Acad. Polon. Sci. Sér. Sci. Math. Astronom. Phys. 26 (1978), 837-840.

10. __ Fans and embeddings in the plane, Pacific J. Math. 83 (1979), 495-503.

11. __ Properties of contractible fans, Doctoral dissertation, Wayne State Univ., Detroit, Mich., 1978.

12. __ Internal characterizations of contractibility for fans, Bull. Acad. Polon. Sci. Sér. Sci. Math. Astronom. Phys. 27 (1979), 391-395.

Department of Mathematics, University of Houston, Houston, Texas 77004

Current address: Department of Mathematics, University of Saskatchewan, Saskatoon, Canada S7N owo 